\title{
Passive transfer of fibromyalgia symptoms from patients to mice
}

\author{
Andreas Goebel, ${ }^{1,2}$ Emerson Krock, ${ }^{3}$ Clive Gentry, ${ }^{4}$ Mathilde R. Israel, ${ }^{4}$ Alexandra Jurczak, ${ }^{3}$ Carlos Morado Urbina, ${ }^{3}$ \\ Katalin Sandor, ${ }^{3}$ Nisha Vastani, ${ }^{4}$ Margot Maurer, ${ }^{4}$ Ulku Cuhadar, ${ }^{4}$ Serena Sensi, ${ }^{2}$ Yuki Nomura, ${ }^{3}$ Joana Menezes, ${ }^{3}$ Azar Baharpoor, ${ }^{3}$ \\ Louisa Brieskorn, ${ }^{3}$ Angelica Sandström, ${ }^{5}$ Jeanette Tour, ${ }^{5}$ Diana Kadetoff, ${ }^{5,6}$ Lisbet Haglund, ${ }^{7}$ Eva Kosek, ${ }^{5,8}$ Stuart Bevan, ${ }^{4}$ \\ Camilla I. Svensson, ${ }^{3}$ and David A. Andersson ${ }^{4}$ \\ ${ }^{1}$ Walton Centre NHS Foundation Trust, Liverpool, United Kingdom. ${ }^{2}$ Pain Research Institute, Institute of Life Course and Medical Sciences, University of Liverpool, Liverpool, United Kingdom. ${ }^{3}$ Department \\ of Physiology and Pharmacology, Karolinska Institutet, Stockholm, Sweden. ${ }^{4}$ King's College London, Wolfson CARD, Institute of Psychiatry, Psychology \& Neuroscience, Guy's Campus, London, United \\ Kingdom. 'Department of Clinical Neuroscience, Karolinska Institutet, Stockholm, Sweden. ${ }^{5}$ Stockholm Spine Center, Upplands Väsby, Sweden. ${ }^{7}$ Department of Surgery, Division of Orthopaedic Surgery, \\ McGill University, Montreal, Quebec, Canada. ${ }^{8}$ Department of Surgical Sciences, Uppsala University, Uppsala, Sweden.
}

\begin{abstract}
Fibromyalgia syndrome (FMS) is characterized by widespread pain and tenderness, and patients typically experience fatigue and emotional distress. The etiology and pathophysiology of fibromyalgia are not fully explained and there are no effective drug treatments. Here we show that IgC from FMS patients produced sensory hypersensitivity by sensitizing nociceptive neurons. Mice treated with IgC from FMS patients displayed increased sensitivity to noxious mechanical and cold stimulation, and nociceptive fibers in skin-nerve preparations from mice treated with FMS IgC displayed an increased responsiveness to cold and mechanical stimulation. These mice also displayed reduced locomotor activity, reduced paw grip strength, and a loss of intraepidermal innervation. In contrast, transfer of IgG-depleted serum from FMS patients or IgG from healthy control subjects had no effect. Patient IgG did not activate naive sensory neurons directly. IgG from FMS patients labeled satellite glial cells and neurons in vivo and in vitro, as well as myelinated fiber tracts and a small number of macrophages and endothelial cells in mouse dorsal root ganglia (DRG), but no cells in the spinal cord. Furthermore, FMS IgG bound to human DRG. Our results demonstrate that IgC from FMS patients produces painful sensory hypersensitivities by sensitizing peripheral nociceptive afferents and suggest that therapies reducing patient IgC titers may be effective for fibromyalgia.
\end{abstract}

\section{Introduction}

Fibromyalgia syndrome (FMS) is a chronic pain condition characterized by widespread pain, augmented pain sensitivity to mechanical pressure and cold temperatures (1-4), as well as fatigue and emotional distress (5-7). The prevalence of FMS is at least $2 \%$ (8), and approximately $80 \%$ of FMS patients are women. The prevalence rises to $10 \%-30 \%$ among patients diagnosed with autoimmune rheumatological conditions $(9,10)$, and FMS is thus one of the most common chronic pain conditions. The etiology and pathophysiology of FMS are not completely understood (11) and the current treatment strategies for FMS rely mainly on lifestyle changes, physical exercise, and drug therapy with antidepressants and anticonvulsants. Unfortunately, the modest efficacy of the available therapies in most patients leaves an enormous unmet clinical need $(12,13)$. The animal models that have been used for experimental studies of FMS have uncertain

Authorship note: AG and E Krock are co-first authors. CIS and DAA are co-senior authors.

Conflict of interest: DAA has received research support from Eli Lilly \& Co. Copyright: (C) 2021, Goebel et al. This is an open access article published under the terms of the Creative Commons Attribution 4.0 International License. Submitted: September 11, 2020; Accepted: May 11, 2021; Published: July 1, 2021 Reference information: J Clin Invest. 2021;131(13):e144201.

https://doi.org/10.1172/JCI144201. translational relevance and rely on local repeated intramuscular injections of acid (14) or systemic depletion of monoamines by reserpine treatment (15). The development of novel, mechanism-based therapies has been hampered by the limited understanding of the basis of FMS.

The increased polymodal pain sensitivity experienced by FMS patients $(1,16)$ is associated with altered pain processing in the central nervous system (11), dysfunctional descending pain modulation $(17,18)$, and structural and functional changes in the brain (19-21). FMS is also associated with abnormalities in peripheral sensory afferents, such as spontaneous activity and sensitization of C-fibers, and loss of epidermal innervation $(22,23)$. Altered levels of inflammatory and immunoregulatory cytokines have also been reported in FMS patients. Although these alterations do not follow a consistent pattern $(24,25)$, they may suggest that immune processes are dysregulated in patients. These observations, together with the markedly increased prevalence of FMS among patients with autoimmune rheumatological conditions $(9,10)$, and demonstrations that less common $(26,27)$ and very rare chronic pains $(28)$ are caused by autoantibodies, led us to hypothesize that FMS may have an autoimmune basis. Here we have investigated the possibility that autoreactive IgG is responsible for several key symptoms of FMS, by examining whether these can be transferred to mice by administration of $\operatorname{IgG}$ purified from FMS patients, and by assessing the tissue localization of IgG after passive transfer. 
A

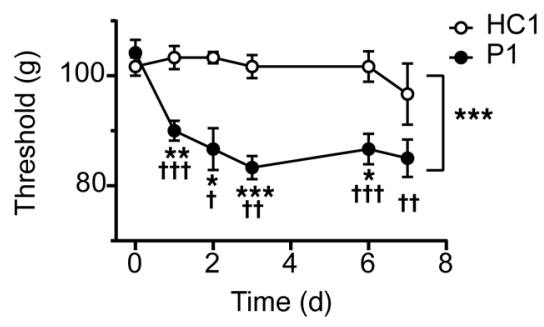

B
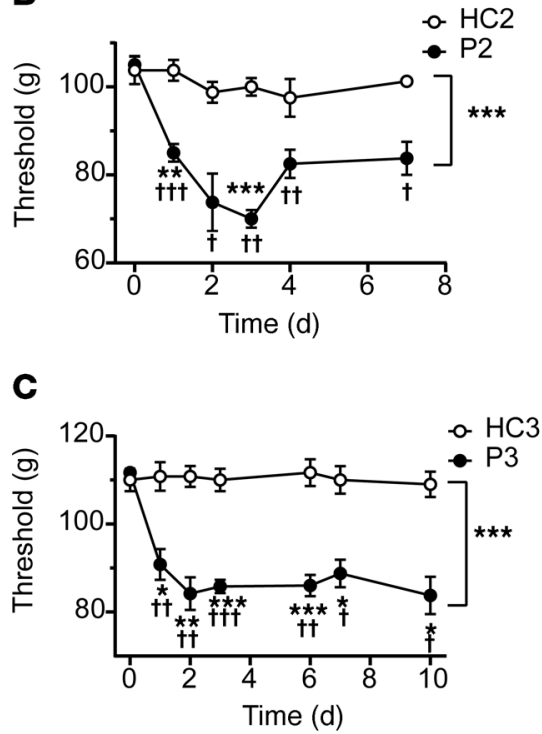

D

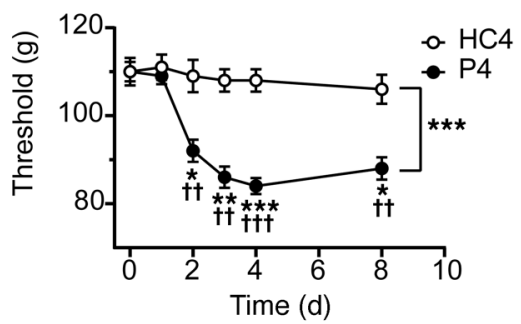

E

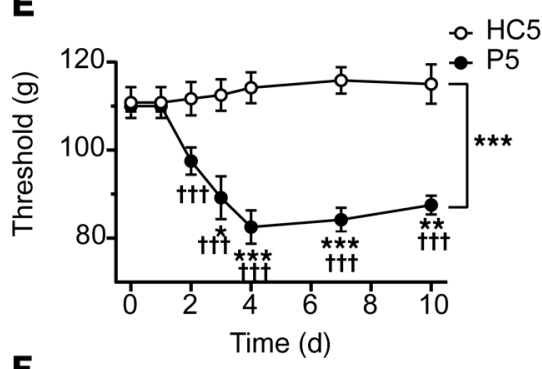

$\mathbf{F}$

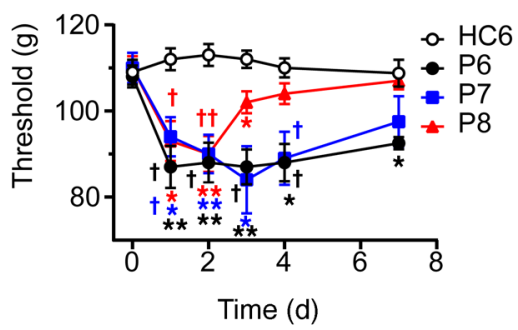

G

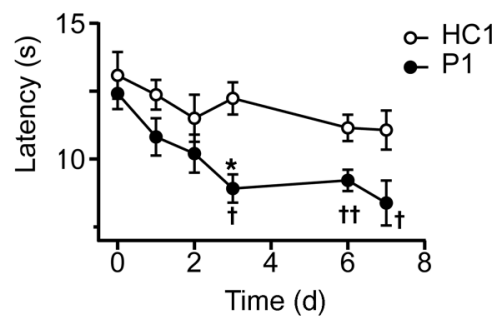

H

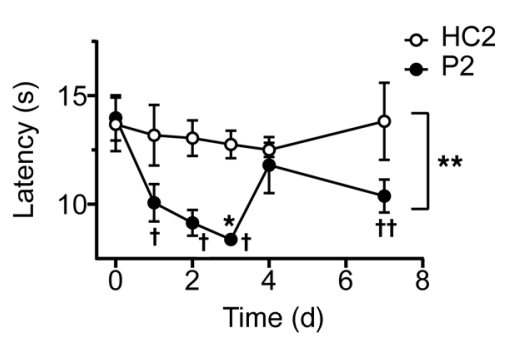

I

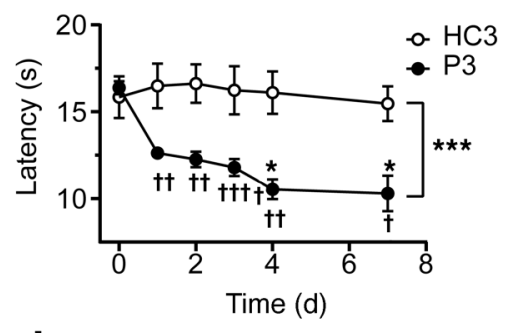

J

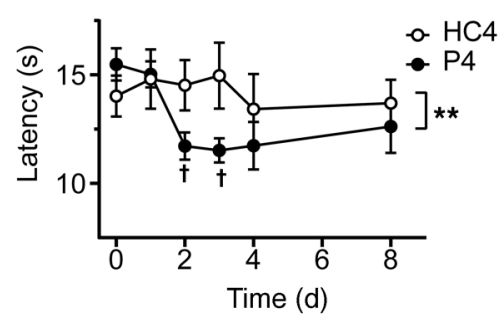

$\mathbf{K}$
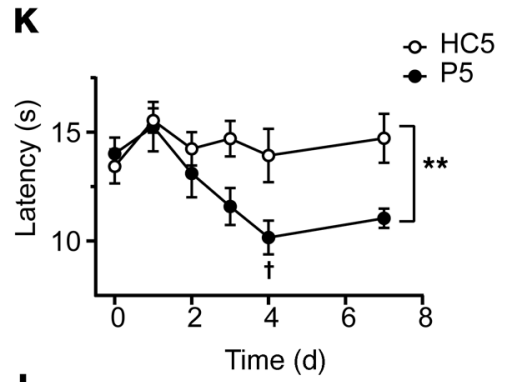

$\mathbf{L}$

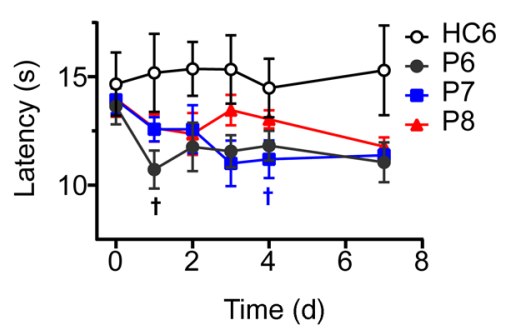

Figure 1. Passive transfer of hypersensitivities from fibromyalgia patients to mice. Administration of IgG (8 mg on 4 consecutive days) from each of 8 different FMS patients (P1-P8) significantly reduced the withdrawal threshold in the paw-pressure test (A-F) compared with IgG from healthy control subjects (HC1-HC6). The paw withdrawal latency in the cold-plate test was reduced by lgG from 7 of 8 patients (G-L). Data points are mean \pm SEM of $n=6$ mice in $\mathbf{A}, \mathbf{C}, \mathbf{E}, \mathbf{G}, \mathbf{I}$, and $\mathbf{K}$; $n=5$ in $\mathbf{D}, \mathbf{F}, \mathbf{J}$, and $\mathbf{L}$; and $n=4$ in $\mathbf{B}$ and $\mathbf{H} .{ }^{*} P<$ $0.05,{ }^{* *} P<0.01,{ }^{* *} P<0.001$, FMS IgG compared with HC IgG; 2-way repeated measure ANOVA followed by Sidak's correction. ${ }^{\dagger} P<0.05,{ }^{\dagger \dagger} P<0.01$, ${ }^{t+t} P<0.001$, compared with the naive preinjection value at time zero; 2-way repeated measure ANOVA followed by Dunnett's test.

\section{Results}

Passive transfer of sensory hypersensitivities. IgG purified from the serum of individual FMS patients and healthy control (HC) subjects recruited from the Walton Centre (Liverpool, United Kingdom [UK]) was administered to female mice by intraperitoneal injection for 4 consecutive days (8 $\mathrm{mg}$ per day). This dose regimen was based on the original studies identifying myasthenia gravis as an autoantibody-mediated disorder $(29,30)$, and more recent studies of complex regional pain syndrome $(26,31)$. Because FMS is characterized by hypersensitivity to mechanical pressure, we examined paw withdrawal thresholds in mice using the Randall-Selitto paw-pressure test following IgG transfer. IgG from each of the 8 individual patients, but not from any of the 6 HC subjects, rapidly produced mechanical hypersensitivity (Figure 1, A-F). In addition to pressure sensitivity, patients frequently report that pain is exacerbated by cold temperatures, and quantitative sensory testing has demonstrated an increased cold pain sensitivity in FMS $(1,2,4,16)$. In good agreement with patient observations, administration of IgG from 7 of the $8 \mathrm{FMS}$ patients gave rise to a significantly increased sensitivity to noxious cold in mice (Figure 1, G-L). Both mechanical and cold hypersensitivities were typically established within 24-48 hours after the first injection and were maintained for more than 1 week. The observed hypersensitivities produced by IgG preparations from different FMS patients showed similar amplitudes and time courses (Figure 1, A-L, and Supplemental Figure 1, A and $\mathrm{B}$; supplemental material available online with this article; https://doi.org/10.1172/ 
A

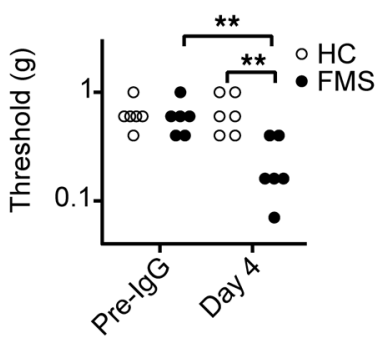

E

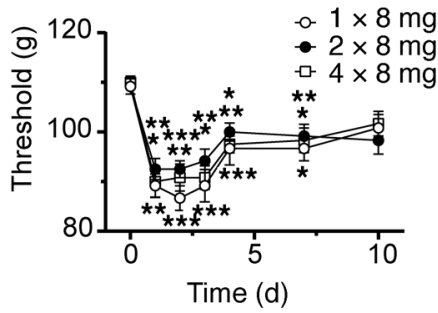

B

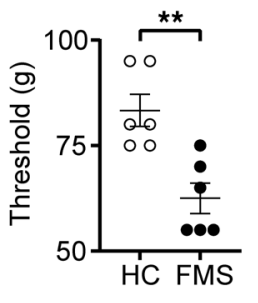

C

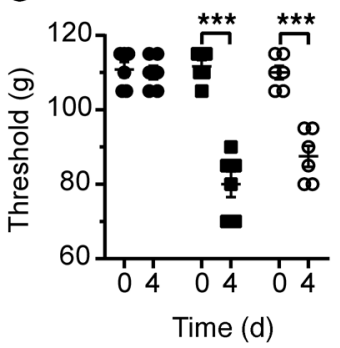

D

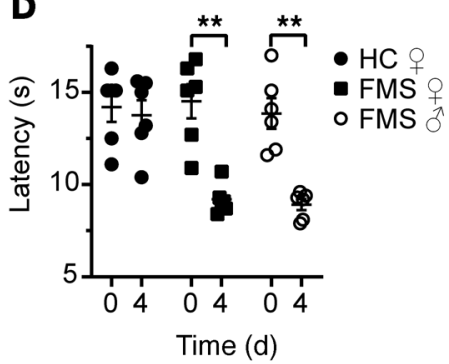

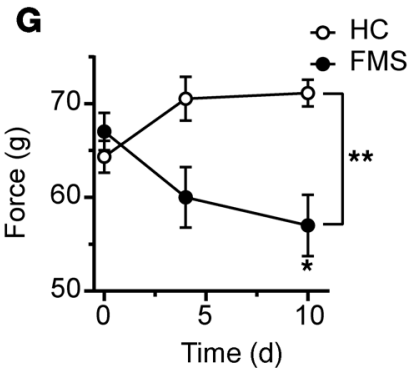

Figure 2. FMS IgG produces polymodal abnormalities. FMS IgG increased the sensitivity to punctate stimulation with von Frey filaments (A). The threshold for leg withdrawal in response to pressure applied to the thigh (using a Randall-Selitto device) was reduced by FMS IgG compared with HC IgC (B). Female and male mice are affected equally by FMS IgG in the paw-pressure test (C) and the cold-plate test (D). Mechanical hypersensitivity produced by either 1, 2, or 4 injections of $8 \mathrm{mg}$ FMS IgG (E), and by single injections of 2, 4, or $8 \mathrm{mg}$ (F). The front paw grip strength is reduced by FMS IgG compared with HC IgG (G). Data points are mean \pm SEM or individual measurements. ${ }^{*} P<0.05,{ }^{*} P<0.01$, ${ }^{* *} P<0.001$, FMS IgG compared with HC IgG; 2 -way repeated measure ANOVA followed by Sidak's correction (A, C, D, and $\mathbf{G}$ ). Data in B were analyzed by unpaired, 2-tailed $t$ test. Data in E and $\mathbf{F}$ were compared to the naive preinjection value at time zero by 2-way repeated measure ANOVA followed by Dunnett's test.

JCI144201DS1), which is reflected in the averaged mechanical and cold sensitivities produced by IgG from these 8 individual FMS patients and $6 \mathrm{HC}$ subjects measured over 10 days (Supplemental Figure 1, C and D). FMS IgG also generated hypersensitivity to stimulation with calibrated von Frey filaments, a widely used test of mechanical nociception in mice (Figure 2A). Fibromyalgia pain is characteristically widespread, and studies of the bodily localization of pain have identified the thigh as one of the most commonly affected sites $(32,33)$. We therefore examined the pressure sensitivity of the thigh using the Randall-Selitto device (Figure 2B), to determine whether FMS IgG affects the pressure sensitivity of sites other than the hind paw in mice. In this test, FMS IgG produced significant mechanical hypersensitivity in the thigh compared with treatment with IgG from HCs (Figure 2B).

FMS is more common among women than men, and we therefore asked whether an increased sensitivity to the effects of IgG contributes to the increased incidence in females. We examined this possibility by comparing the effects of IgG from a female patient in female and male mice. We observed significant and indistinguishable mechanical and cold hypersensitivities in female and male mice (Figure 2, C and D), suggesting that it is unlikely that the described behavioral findings are due to increased female sensitivity to FMS IgG.

Hitherto, we had administered 4 injections of $8 \mathrm{mg}$ IgG in all experiments. To identify a dose-response relationship for IgG from one patient, we varied the number of daily injections between 1 and 4 (Figure $2 \mathrm{E}$ ). In this experiment, 1,2 , and 4 injections of $8 \mathrm{mg}$ produced essentially identical patterns of mechanical hypersensitivity. We additionally examined the effects of single injections of
2, 4, and $8 \mathrm{mg}$ of FMS IgG, and found that only the highest dose increased the mechanical sensitivity in the paw-pressure test (Figure $2 \mathrm{~F}$ ). These data suggest that a single injection of $8 \mathrm{mg}$ IgG may be sufficient to transfer mechanical hypersensitivity to mice, and that the effect is saturable (Figure 2E).

Since FMS is regularly associated with reduced muscular strength, and FMS severity is negatively correlated with handgrip strength $(34,35)$, we sought to evaluate this experimentally by monitoring the front paw grip strength in mice. In this test, FMS IgG significantly reduced grip strength compared with mice treated with HC IgG (Figure 2G).

To determine whether antibodies are responsible for painful hypersensitivities in a larger and regionally distinct cohort of FMS patients (recruited at the Karolinska Institute; Supplemental Table 1), we examined the effects of IgG preparations pooled from multiple FMS patients and HC subjects. As expected, FMS patients displayed markedly higher pain ratings on a visual analog scale (VAS) (Figure 3A and Supplemental Table 2) and a significantly increased pressure-pain sensitivity compared with HC subjects (Figure 3B and Supplemental Table 2). Serum IgG isotype levels of FMS and HC subjects were within published normal concentration ranges (36) despite IgG1 being lower in FMS serum than in HC serum (for Swedish subjects; Supplemental Table 3). Administration of $\operatorname{IgG}$ pooled from 2 separate groups of FMS patients to mice (8-14 individuals per group, 2 experiments; Supplemental Table 2) significantly increased sensitivity in the paw-pressure test compared with administration of pooled IgG from groups of $\mathrm{HC}$ subjects (Figure $3 \mathrm{C}$ ). In these experiments, we used a dose regimen of $8 \mathrm{mg} \operatorname{IgG}$ on 4 consecutive days. Similar to our observa- 
A
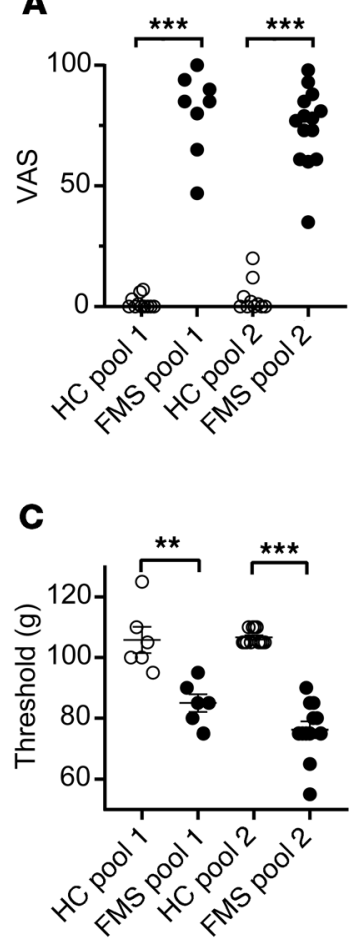

E

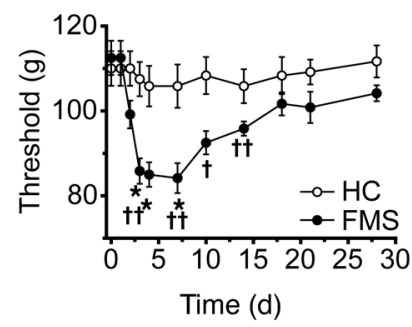

B

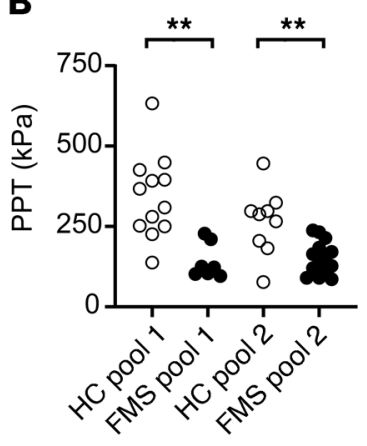

D

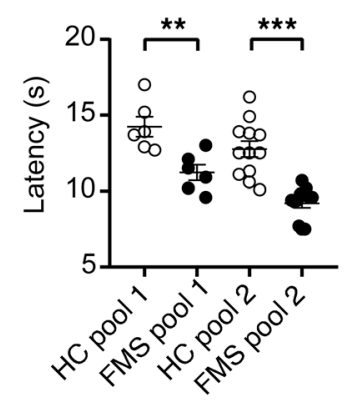

$\mathbf{F}$

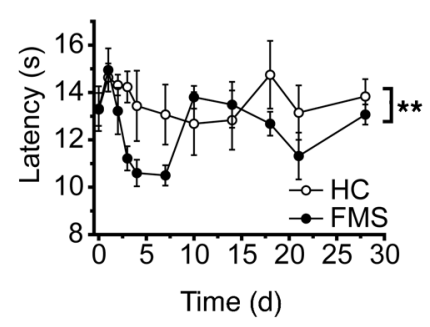

Figure 3. Passive transfer of hypersensitivity by IgG pooled from multiple patients. Visual analog pain scores (VAS, A) and pressure-pain thresholds (PPT, B) in 2 pools of FMS patients and healthy control (HC) subjects. Pool 1, $n=8$ FMS and $n=12 \mathrm{HC}$; Pool 2, $n=14 \mathrm{FMS}$ and $n=10 \mathrm{HC}$. ${ }^{*} P<$ $0.01,{ }^{* *} P<0.001$ by Mann-Whitney $U$ test. Administration of IgG pooled from FMS patients produced mechanical (C) and cold (D) hypersensitivity in mice compared with pooled $\mathrm{HC}$ IgG, 4 days after the first injection. Pool $1, n=6$ mice per group; Pool 2, $n=12$; line and whiskers indicate mean \pm SEM. ${ }^{* *} P<0.01,{ }^{* *} P<0.001$ by unpaired, 2 -tailed $t$ test. Time course of mechanical $(\mathbf{E})$ and cold $(\mathbf{F})$ hypersensitivity following administration of pooled IgG (Pool 1). ${ }^{*} P<0.05$, FM vs. HC IgG; 2-way repeated measure ANOVA with Sidak's correction. ${ }^{\dagger} P<0.05,{ }^{\dagger \dagger} P<0.01$, compared with day zero; 2-way repeated measure ANOVA followed by Dunnett's test. Data in $\mathbf{E}$ and $\mathbf{F}$ are mean \pm SEM of 6 mice per group.

tions with IgG from individual patients (Figure 1), the pooled FMS IgG also elicited hypersensitivity to cold (Figure 3D). To determine the duration of the IgG-mediated hypersensitivity in more detail, we studied mechanical thresholds and cold latencies in mice treated with IgG pooled from patients or HC subjects for 1 month. The onset of hypersensitivities produced by pooled FMS IgG followed the same time course as seen with IgG from individual donors and resolved fully about 2.5 weeks after cessation of IgG administra-

tion (Figure 3, E and F; see Supplemental Figure 1 for time course with $\operatorname{IgG}$ from an individual patient), which is consistent with the time course for the elimination of human IgG from mice (37).

We next compared the effects of FMS patient IgG with the IgG-depleted patient serum from the same individual to assess whether immunoglobulins and serum components other than $\operatorname{IgG}$ may contribute to pain and hypersensitivity (Figure 4). As expected, IgG from an FMS patient (8 mg on 4 consecutive days) produced marked cold and mechanical hypersensitivities, but IgG-depleted serum from the same patient and pooled preparations from groups of FMS patients or HC subjects were without any discernable behavioral effect (Figure $4, \mathrm{~A}$ and $\mathrm{B}$ ).

IgG from patients reduces locomotor activity in mice. Fatigue, a feeling of excessive mental or physical weariness and weakness, is one of the key symptoms of FMS (38), but also of a wide range of other chronic diseases (39). FMS patients often display reduced levels of physical activity (40), with a loss of physical fitness as a consequence (41). Encouraged by the demonstration of reduced grip strength following administration of FMS IgG, we next examined locomotor activity of mice injected with FMS IgG or HC IgG over a 24-hour period. In this experiment we used pooled IgG from new groups of FMS patients and HC subjects (Pool 3, Supplemental Table 2). Similar to the earlier 2 cohorts (see Figure 3, A and B), patients displayed higher VAS pain ratings and increased pressure-pain sensitivity compared with HC subjects (Figure 5, A and B, and Supplemental Table 2). Since the dose-response studies showed that 2 injections of FMS IgG were sufficient to induce marked mechanical hypersensitivity (see Figure 2), $8 \mathrm{mg}$ of pooled IgG was injected intraperitoneally on 2 consecutive days. As expected, mice were markedly less active during the day than during the dark phase (18:00-6:00 hours), irrespective of whether they received HC or FMS IgG (Figure 5C). During the night (18:00-6:00 hours, when mice are most active), the total number of recorded movements in FMS IgG-treated mice was reduced. When examined in more detail, it was apparent that this reduction was predominantly in the most active phase (22:00-02:00 hours; Figure 5, D-F). These results demonstrate that FMS IgG negatively impacts evoked and nonevoked behaviors in mice.

Sensitization of nociceptors. Microneurography of FMS patients has demonstrated sensitization of nociceptive C-fibers (22). To determine whether the sensory abnormalities demonstrated in mice in vivo following administration of FMS patient IgG could be explained by a sensitization of peripheral nociceptors, we examined single afferent fiber units in skin-saphenous nerve preparations from IgG-treated mice. Single units were classified according to their conduction velocity and mechanical response threshold, as described previously $(42,43)$.

We stimulated the receptive fields of $\mathrm{A} \delta$ - and C-mechanosensitive nociceptors (AM and CM fibers) mechanically and compared the mechanical activation thresholds (the force required to elicit at least 2 action potentials during a 2-second challenge) after 4 days of treatment with HC or FMS IgG (Figure $6, \mathrm{~A}-\mathrm{C}$ ). Both $\mathrm{AM}$ and $\mathrm{CM}$ fibers in preparations from mice treated with FMS IgG responded to mechanical stimulation at a reduced force compared with preparations from $\mathrm{HC}$ IgG-treated mice (Figure 6, A-C). The reduced mechanical response thresholds are consistent with the $\mathrm{C}$-fiber sensitization observed in 
A

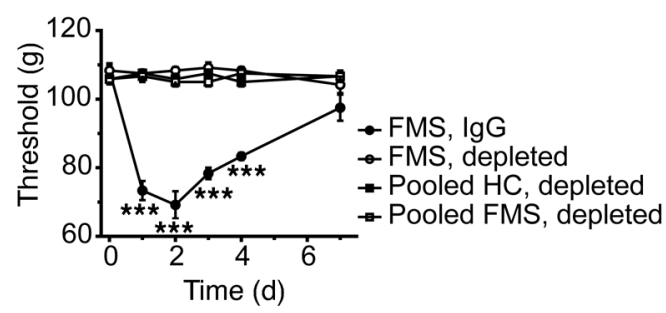

B

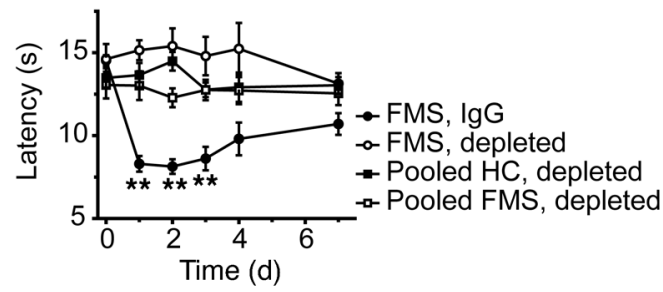

Figure 4. IgG-depleted serum is inactive. IgG from an FMS patient, but not IgG-depleted serum from the same patient or pooled from FMS or $\mathrm{HC}$ cohorts, produced mechanical (A) and cold (B) hypersensitivities. ${ }^{* *} P<$ $0.01,{ }^{* *} P<0.01$, FM vs. HC IgG; 2-way repeated measure ANOVA followed by Sidak's correction. Data points are mean \pm SEM of 6 mice per group.

FMS patients using microneurography (22) and demonstrate that patient IgG produces a heightened peripheral nociceptor responsiveness that is maintained in the absence of the central nervous system. Since we had observed a robust behavioral cold hypersensitivity in mice treated with FMS IgG, we also examined whether this phenotype was accompanied by an increased cold responsiveness in mechanosensitive C-fibers (Figure 6, $\mathrm{D}-\mathrm{F})$. We challenged $\mathrm{CM}$ fibers with a 60 -second cooling ramp (from $32^{\circ} \mathrm{C}$ to $5^{\circ} \mathrm{C}-9^{\circ} \mathrm{C}$ ) and noted that a larger proportion of $\mathrm{CM}$ fibers responded to cold in preparations from mice treated with FMS IgG compared with HC IgG (Figure 6D). In contrast, FMS IgG did not influence the cold activation thresholds of coldsensitive CM (Figure 6E). An increasing number of human disorders are recognized as produced by functional autoantibodies that alter excitability by binding to neuronal surface proteins (44). We therefore challenged isolated neurons directly with a high concentration of FMS IgG $(200 \mu \mathrm{g} / \mathrm{mL})$, to determine whether FMS IgG directly excites dorsal root ganglia (DRG) neurons by such an interaction. We observed no effect of FMS IgG on intracellular $\mathrm{Ca}^{2+}$ concentration $\left(\left[\mathrm{Ca}^{2+}\right]_{i}\right)$ in any of the 870 neurons examined (Figure 6G).

In vivo immunohistochemical localization of human IgG. Since $\mathrm{CM}$ and AM fibers were hypersensitive in mice treated with FMS IgG, we investigated the localization of FMS IgG to better understand potential sites of action. In mice injected with pooled IgG, FMS IgG was consistently detected in DRG, but neither in brain nor spinal cord tissue by Western blot analysis (Figure 7A; see complete unedited blots in the supplemental material). Immunohistochemical analysis of tissues from mice that had been injected with pooled FMS IgG, using anti-human IgG antibodies to detect FMS IgG, revealed robust staining in the lumbar DRG (Figure 7, B and $C$ ), while no specific immunoreactivity was observed in the spinal cord (Figure 7A and Supplemental Figure 2A). The percentage area that was immunoreactive for human $\operatorname{Ig} G$ as well as the pixel intensity were much greater in mice that had been injected with FMS IgG compared with IgG from HC subjects, and antihuman IgG generated only minimal reactivity in DRG from salineinjected mice (Figure 7, B and C).

FMS patient IgG staining was primarily localized to satellite glial cells (SGCs, visualized by glutamine synthase staining) and to fiber tracts entering the DRG, as well as a small number of Iba1positive macrophages and CD31-positive blood vessels (Figure 7D). FMS IgG did not appear to colocalize with the neuronal nuclear marker NeuN, which labels neuronal nuclei and to a lesser extent cytoplasm. Since NeuN does not label neuronal membranes and SGCs enwrap neurons with as little as $20 \mathrm{~nm}$ separation between the cells, it was challenging to distinguish neuronal membrane staining from that of the surrounding SGC (Figure 7D). We further explored the possibility that FMS IgG also labels the membranes of sensory neurons by using TrkA as a neuronal surface marker. In addition to the extensive FMS IgG labeling of glutamine synthaseexpressing SGCs (Figure 7, D and E), FMS IgG also appeared to label some, but not all, TrkA-expressing neurons (Figure 7E).

FMS IgG promotes expression of SGC activity markers. Since FMS IgG frequently colocalized with SGCs, we investigated whether this was associated with evidence of changes in SGC activity. We found that the positive area and pixel intensity of glial fibrillary acidic protein (GFAP) immunoreactivity (Figure 8, A and B) and Gfap and s100b gene expression (Figure 8C) in DRG from mice injected with FMS IgG were increased, compared with HC IgG-injected controls. These changes suggest that the presence of FMS IgG in DRG had increased the activity of SGCs. In contrast, the Iba1 percentage area of immunoreactivity in the neuron-rich area of the DRG was not different between FMS IgG- and HC IgG-injected mice (Figure 8, D and E), suggesting that FMS IgG accumulation in the DRG did not stimulate local macrophage proliferation or infiltration. However, Aifi (encoding Iba1) and Itagm (encoding CD11b) were both elevated in the DRG of FMS IgG-injected mice (Figure 8F), indicating that certain aspects of macrophage activity were altered by FMS IgG. Importantly, there were few to no apoptotic cells in the DRG of mice injected with FMS or HC IgG (Supplemental Figure 3A), indicating that the effects of FMS IgG are not due to neuronal cell death. In contrast to the DRG, no changes in astrocyte and microglia reactivity were observed in the spinal cord based on GFAP (Supplemental Figure 2, B-D) and Iba1 (Supplemental Figure 2, E-G) immunoreactivity, respectively. Together, these results suggest that FMS IgG has a local effect in the DRG.

FMS IgG does not induce cytokine production or systemic inflammation. Although there are inconsistencies between published studies, levels of inflammatory and immunomodulatory cytokines and chemokines, such as IL-6, IL-8, and IL-10, have been reported to be elevated in FMS serum or plasma (22, 23) compared with HCs. Thus, we assessed a panel of factors in serum from mice injected with FMS IgG, HC IgG, or saline. IL-1 $\beta$ was below the limit of detection in 5 of 6 samples in each group, indicating that neither FMS IgG nor HC IgG induced IL-1 $\beta$ production. Similarly, there was no difference in TNF- $\alpha$, CXCL1 (a murine analog of human IL-8), IL-2, IL-5, IL-6, IL-10, and IFN- $\gamma$ levels between the groups (Figure 8, G-M). These data demonstrate that FMS IgG injection does not alter sys- 
A

C
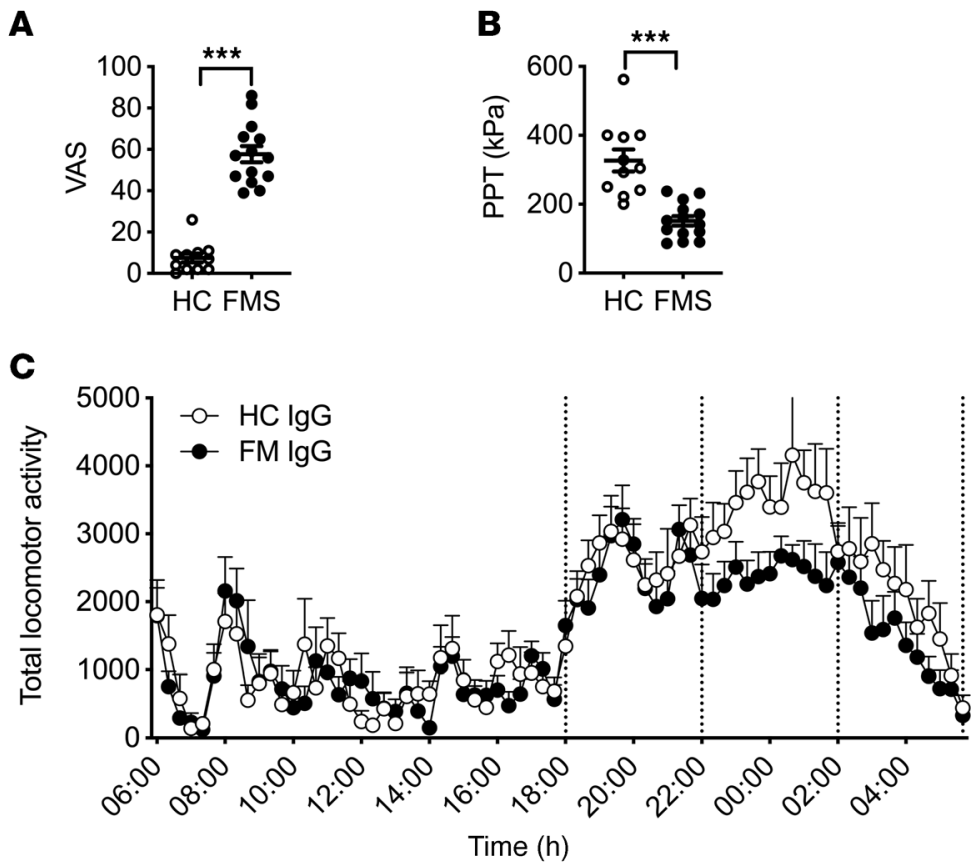

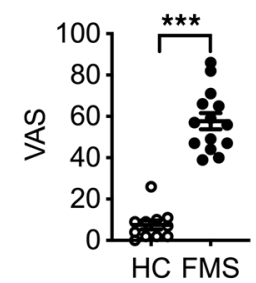

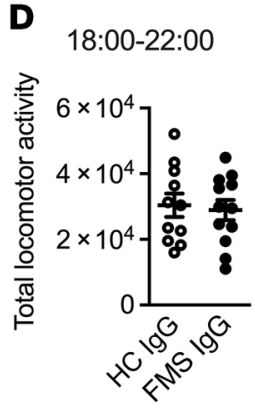

$\mathbf{E}$
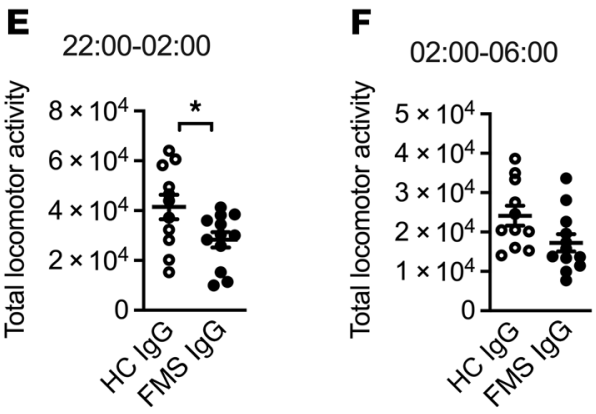

Figure 5. Passive transfer of FMS IgC decreases locomotor activity. Visual analog pain scores (VAS, A) and pressure-pain thresholds (PPT, B) reported by FMS patients and healthy control (HC) subjects in Pool 3. Following transfer of HC or FMS IgC to mice, locomotor activity was assessed over a 24-hour period using a Comprehensive Animal Lab Monitoring System. The total number of recorded movements was similar between HC IgG and FMS IgG mice during the day phase (low activity), but FMS IgG-injected mice showed less activity during the night phase (high activity) (C). The night phase was divided into 3 phases: 18:00-22:00 hours (D), 22:00-02:00 hours (E), and 02:00-06:00 hours (F), indicated by the dotted lines. Mice injected with FMS IgC displayed significantly less locomotor activity during the peak activity phase (E) compared with $\mathrm{HC}$ IgG-injected mice. Data points are mean $\pm \mathrm{SEM}$, FMS $n=14, \mathrm{HC} n=11$, $n=12$ mice per group. ${ }^{*} P<0.05$, ${ }^{* *} P<0.001$ by Mann-Whitney $U$ test $(\mathbf{A}$ and $\mathbf{B})$, 2-way ANOVA followed by Bonferroni's correction (C), or unpaired $t$ test (D-F). neurons. FMS IgG bound to a significantly larger proportion of cells (290 of 368 cells analyzed, $79 \%$ ) compared with HC IgG (84 of 264 cells analyzed, 32\%) (Figure $9, \mathrm{~A}-\mathrm{C}$ ), and recognized these cells more strongly, as judged by pixel intensity (Figure 9D). Similarly, FMS IgG labeled a significantly larger proportion of both glutamine synthase-positive (SGCs) and -negative cells (nonSGCs) in these cultures and did so with a greater pixel intensity compared with HC IgG (Figure 9, E and F).

In dissociated DRG neuron cultures approximately $30 \%$ of cells were $\beta$ III-tubulin-positive neurons (Figure 9G). Similar to our observations with SGC-enriched cultures, FMS IgG bound to a larger proportion of cells from dissociated DRG (358 of 527 cells analyzed, 68\%) compared with HC IgG (158 of 469 cells analyzed, 34\%), and it also labeled these cells more intensely (Figure 9, H-J). Importantly, FMS IgG labeled almost all BIII-tubulinpositive neurons, whereas HC IgG labeled far fewer (Figure 9K). The pixel intensity of FMS IgG binding to neurons was greater than FMS IgG binding to non-neuronal cells, and greater than $\mathrm{HC}$ IgG binding to neurons and non-neuronal cells (Figure 9L). Neither FMS IgG nor HC IgG affected cell viability compared with cells that were not incubated with antibodies (Supplemental Figure 3, B-E). Finally, incubating live cultures with IB4, a membrane marker of a subpopulation of nociceptors, demonstrated that FMS IgG binding colocalized with neuronal membranes (Figure 9M). In conclusion, FMS IgG binds SGCs in vivo, and while further studies are warranted to determine if FMS IgG also binds to neuronal membranes in vivo, this possibility is supported by our data showing that FMS IgG binds both SGCs and neurons in vitro.

Reduced epidermal innervation. An involvement of peripheral sensory nerves in FMS has previously been indicated by observations of small-fiber pathology in patients $(23,45)$. Importantly, the symptom severity of FMS is correlated with the extent of small-fiber pathology (33). We therefore examined the impact of IgG on intraepidermal nerve fiber density (IENFD) in the glabrous hind paw skin of mice. A significant reduction in IENFD was apparent 14 days after administration of FMS IgG was initiated, compared with skin sections from mice similarly treated with HC IgG (Figure 10, A and B).

Screening FMS IgG reactivity with human protein fragments. To investigate whether FMS IgG from different temic inflammatory and immunomodulatory cytokine levels. Together, the results suggest that FMS IgG acts by generating a local effect in the DRG.

In vitro immunohistochemical binding of FMS IgG. To further differentiate between labeling of SGCs and sensory neurons, we examined the immunoreactivity of FMS and HC IgG in SGCenriched, sensory neuron-depleted cell cultures and dissociated DRG neuron-rich cell cultures. FMS IgG or HC IgG (pooled IgG, $100 \mu \mathrm{g} / \mathrm{mL}$ ) was added to the culture media prior to cell fixation in order to examine membrane labeling in a nonpermeabilizing condition. In SGC-enriched cultures, approximately $75 \%$ of cells were glutamine synthase-positive SGCs and less than $0.5 \%$ were patients contains a common pattern of autoreactivity, we used a proteome-wide microarray screen (SciLifeLab). This assay uses 42,000 human peptides (from $>18,000$ proteins) from the Human Protein Atlas $(46,47)$. The peptides in the array are linear, produced in E. coli, and most are between 50 and 150 amino acids in length. We examined the reactivity of 4 samples, each prepared from equal parts of $4 \mathrm{FMS}$ patients' sera $(4 \times 4)$, and noted that all 4 samples recognized multiple peptides (signal $>$ mean fluorescence intensity $+[4 \times$ SD]; Supplemental Table 5). However, our analysis also revealed that very few peptides were recognized by more than one of the pooled samples, strongly indicating that the antigens included in the array are unlikely to represent a common 
A

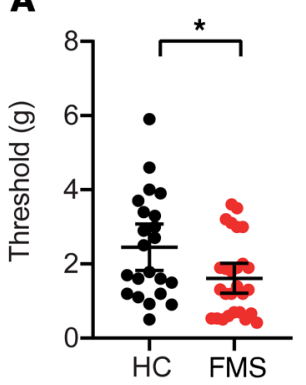

D

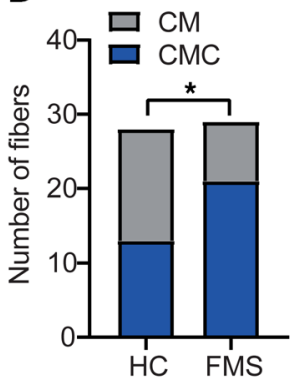

G

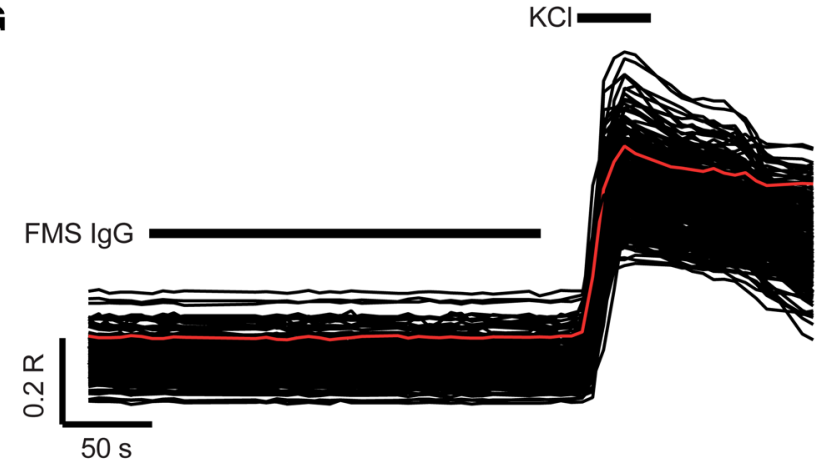

Figure 6. Passive transfer of FMS IgG sensitizes nociceptors. The mechanical activation thresholds of (A) A $\delta$ - (AM) and (B) C-mechanonociceptors (CM) were reduced in preparations from mice treated with FMS compared with HC IgG ( $n=22-27$ single units). ${ }^{*} P<0.05$ by 1 -tailed Mann-Whitney $U$ test. (C) The example trace illustrates a mechanical threshold response (evoked by the minimum force required to elicit at least 2 spikes) in a CM unit. (D) The proportion of cold-sensitive CM units (CMCs) was increased in preparations from mice treated with FMS IgC (21 of 29 units responded to cold) compared with HC IgG (13 of 28 units responded to cold). ${ }^{*} P<0.05$ by 1 -sided Fisher's exact test. (E) The cold-activation thresholds of CMC fibers did not differ between FMS and HC preparations $(n=$ 13-21). $P>0.05$ by 1-tailed $t$ test. (F) The example trace illustrates a cold-evoked response in a CMC fiber. (G) Application of FMS IgG $(200 \mu \mathrm{g} / \mathrm{mL})$ to isolated DRG neurons loaded with Fura-2 was without effect on $\left[\mathrm{Ca}^{2+}\right]_{i}$ in all 870 examined neurons (identified by their response to $50 \mathrm{mM} \mathrm{KCl}$ ).

The red trace illustrates the average time course of the displayed 230 neurons.
B

E
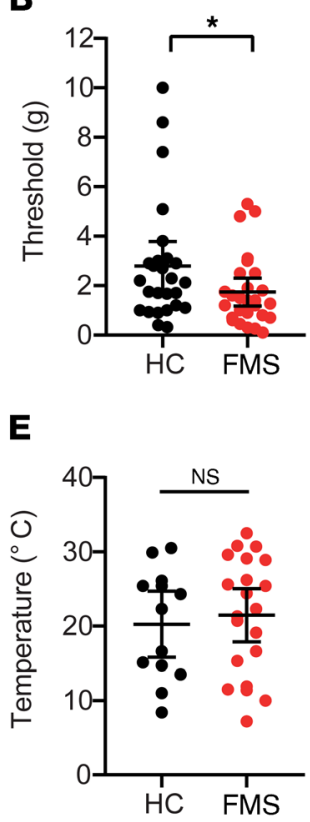

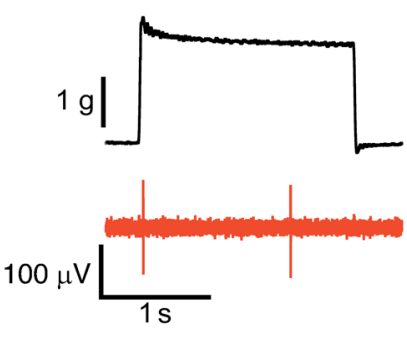

$\mathbf{F}$

$20^{\circ} \mathrm{C}$

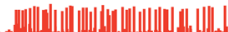

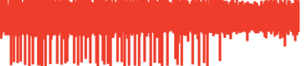

$100 \mu \mathrm{V} \frac{\bigsqcup}{10 \mathrm{~s}}$

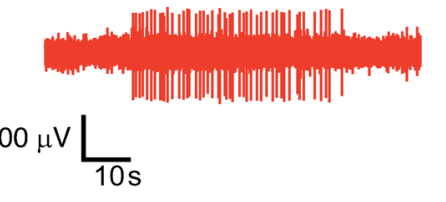

pixel intensity (Figure 11, A and B). Furthermore, FMS IgG bound GFAP-immunoreactive cells and NF200immunoreactive cells, indicating that FMS IgG binds to both human SGCs and human sensory neurons (Figure 11C). FMS IgG also labeled other cells, but less intensely. Taken together, these results suggest that FMS IgG contains autoreactive antibodies that bind antigens expressed in the DRG.

\section{Discussion}

Here we demonstrate that several key features of FMS can be induced in mice by IgG from individuals with FMS. Transfer of hypersensitivities from patients to mice was reproducible across all tested FMS subjects, strongly suggesting that antibody-dependent processes typically underpin the characteristic tenderness and thermal hypersensitivities experienced by patients. The pronociceptive effect of pooled IgG shows that samples from different patients have additive activity, since the IgG from each donor in these preparations was diluted to levels below those required to generate hypersensitivity in mice. The behavioral hypersensitivities produced by FMS IgG were reversible in mice and typically resolved after 2-3 weeks when the levels of human $\operatorname{IgG}$ have decreased (37). The reduction in grip strength and spontaneous locomotor activity, and the reduced density of epidermal innervation observed in mice treated with FMS IgG confirm that in addition to painful hypersensitivities, FMS IgG also produces several non-evoked symptoms and signs of FMS. IgG-depleted serum preparations were without any influence on mechanical or cold nociception, confirming that IgG is the principal pronociceptive, pathological serum component in FMS patients. Importantly, FMS IgG binds to SGCs and neurons in mouse and human

autoantigen in FMS patients. Overrepresentation analysis (http:// webgestalt.org/; ref. 48) suggested some degree of enrichment for proteins associated with Gene Ontology (GO) terms related to cellular components and microtubules (Supplemental Table 6 and Supplemental Figure 4), and a small number of KEGG pathways related to metabolism and vesicle cycling (Supplemental Table 7 and Supplemental Figure 5).

FMS IgG binds to human DRG. Finally, we assessed whether FMS IgG binds cells in human DRG. Human DRG sections were incubated with either pooled HC IgG or FMS IgG and the intensity and localization of binding were analyzed. FMS IgG bound cells in human DRG to a greater extent than HC IgG, as assessed by
DRG, strongly suggesting that FMS patients have autoreactive antibodies.

Interactions between the nervous and immune systems, and in particular autoimmune mechanisms, have increasingly attracted attention in studies of the pathophysiology of chronic pain conditions $(49,50)$. Pronociceptive effects of autoantibodies have been implicated in the uncommon posttraumatic condition complex regional pain syndrome $(31,51)$, where passive transfer of IgG additionally requires an experimental trauma to exert its pronociceptive effects, unlike the observations presented here for FMS IgG. In the very rare, frequently painful neurological condition neuromyotonia, autoantibodies against contactin-associated 
A

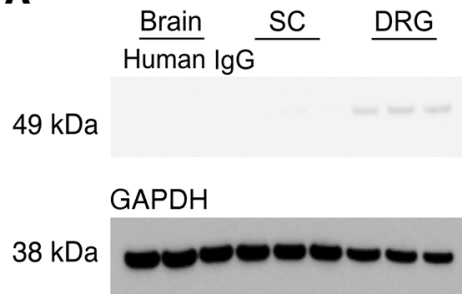

$\mathbf{B}$

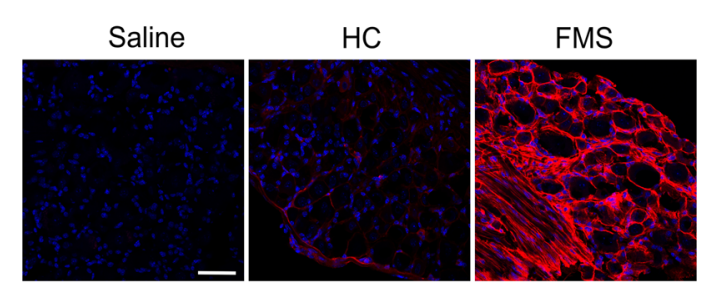

C

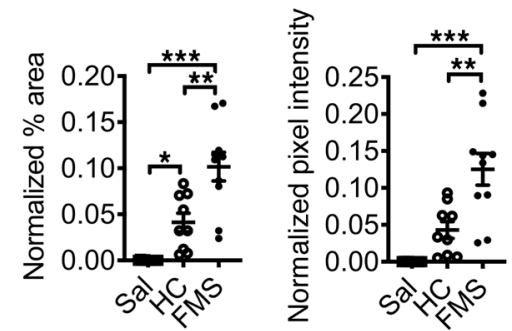

D
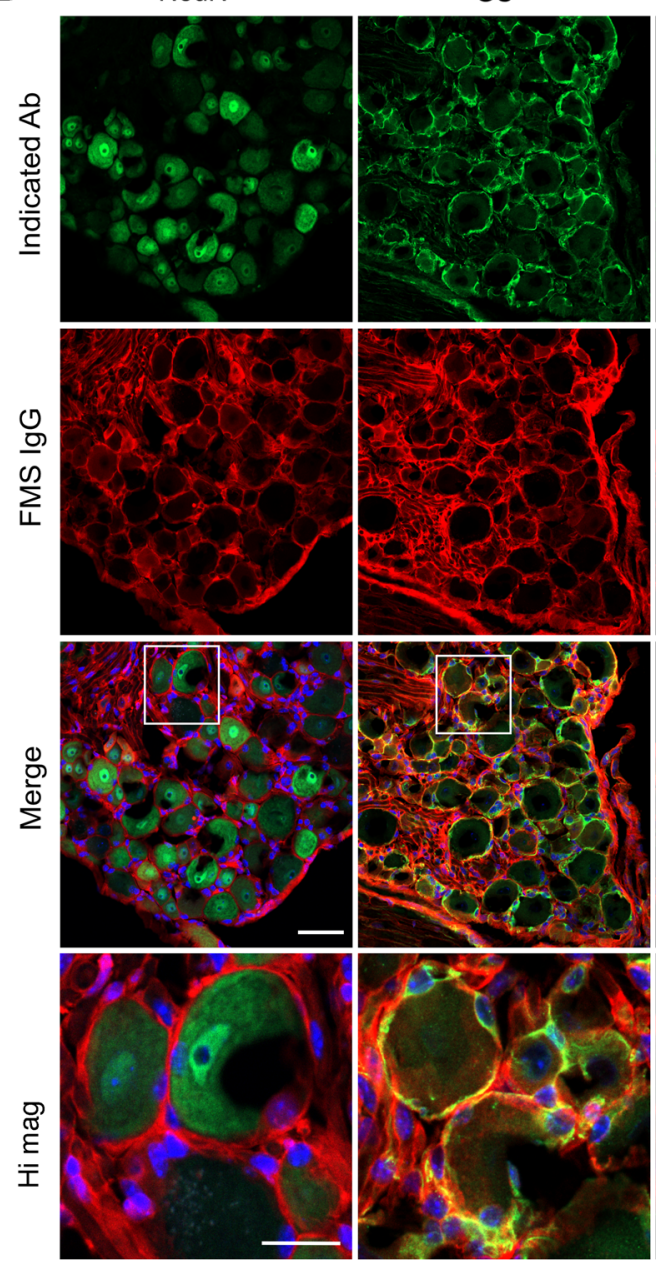

E

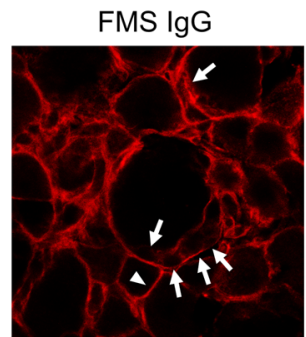

GS

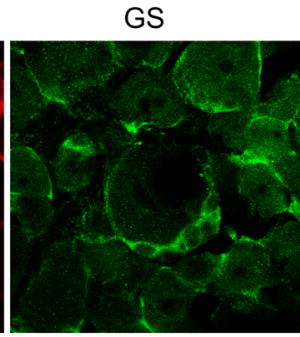

Iba1

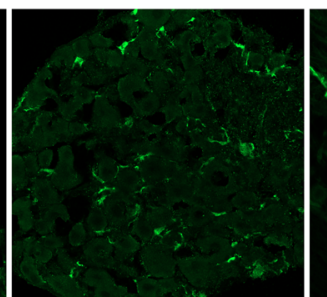

CD31
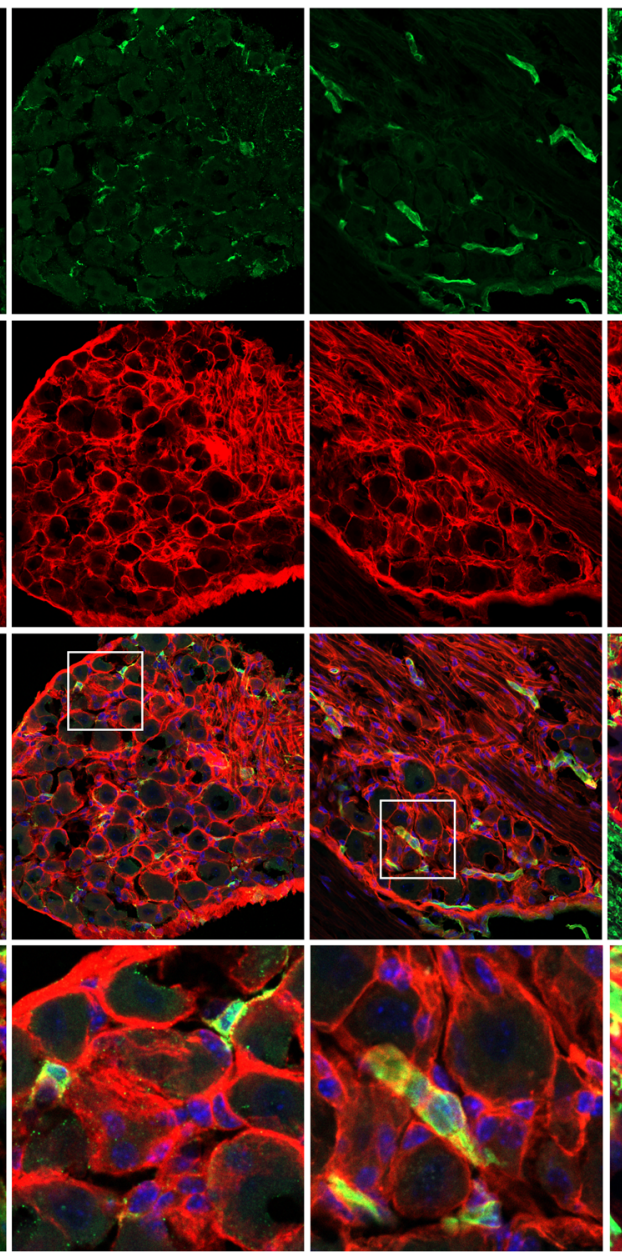

TrkA
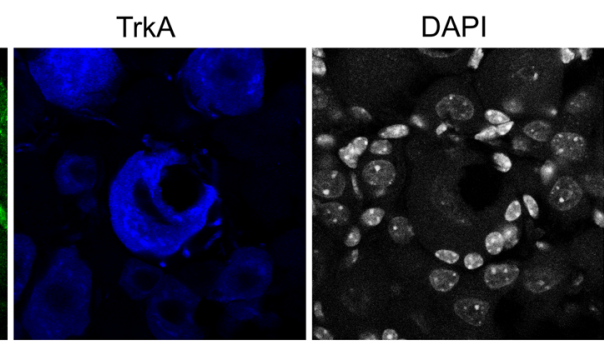

MBP

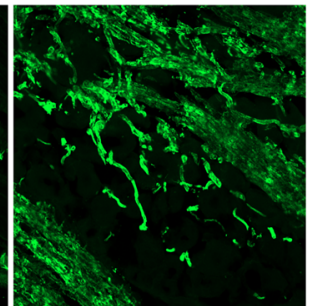

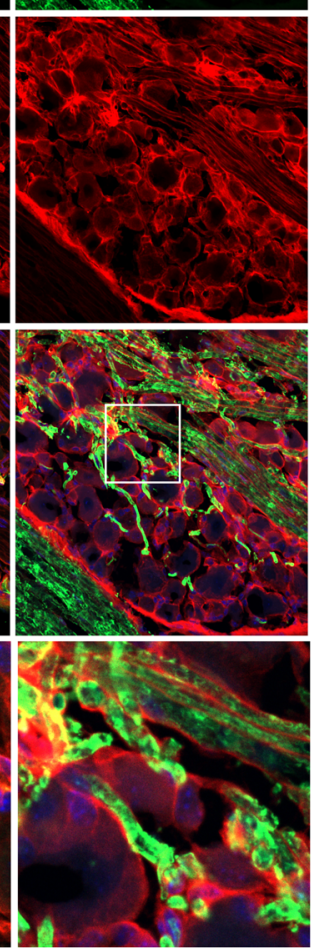


Figure 7. FMS IgG accumulates in the DRG and binds satellite glial cells. Following IgC injection into mice, Western blot analysis detected FMS IgC in the DRG but little to no IgG in spinal cords (SC) or brains (A) (pooled IgG, $8 \mathrm{mg}$ per day for 4 consecutive days, tissue collected after last injection). FMS IgG, but not HC IgG, accumulates in the DRG 14 days after the first IgC injection (B and $\mathbf{C}$ ). Human IgG is red and DAPI is blue. Human IgG immunoreactivity in the neuron-rich area was quantified by assessing the percentage area that was immunoreactive for human IgG and the mean pixel intensity of human IgG. Percentage area and pixel intensity were normalized to the DAPI signal $(n=9-10$; data points are median $\pm 95 \% \mathrm{CI})$. ${ }^{* *} P<0.01,{ }^{* * *} P<0.001$ by 1-way ANOVA followed by Tukey's post hoc test. FMS IgC immunoreactivity does not colocalize with neuronal NeuN staining but does colocalize with satellite glial cells (SGCs) (glutamate synthase-expressing [CS-expressing] cells), some macrophages (Iba1expressing cells) and blood vessels (CD31-expressing cells), and myelinated fiber tracts (myelin basic protein [MBP] staining), but not to myelinated fibers in the DRG (D). To further delineate between SGCs and neuronal membranes, FMS IgG immunoreactivity colocalization was compared with CS and TrkA (a membrane receptor expressed by a subset of nociceptors). FMS IgG colocalizes with GS-expressing SGCs (white arrows) but may also infrequently bind to TrkA-positive neuronal cell membranes (white triangles) (E). Scale bars indicate $50 \mu \mathrm{m}$, except the high-magnification image scale bar and scale bar in $\mathbf{E}$, which indicate $25 \mu \mathrm{m}$.

protein-like 2 (CASPR2) are commonly detected (28). Passive transfer of IgG from these patients produces painful hypersensitivities by internalizing voltage-gated $\mathrm{K}^{+}$channels. Our findings indicate that similarly nondestructive processes produce hypersensitivities, motor symptoms, and loss of epidermal innervation in the common condition FMS.

The importance of altered central nervous system function and plasticity in FMS is illustrated by altered brain activity patterns (19), glia activation in the brain (52), impaired conditioned pain modulation (53), and multisensory hypersensitivity to a wide range of stimuli $(1,54,55)$. However, it is becoming increasingly clear that peripheral alterations also contribute to the underlying pathology, as exemplified by identification of small-fiber pathology in FMS patients (33) and altered cytokine levels (24). Here we found that mechanosensitive nociceptors in skin-nerve preparations from FMS IgG-treated mice displayed an increased responsiveness to mechanical and cold stimuli, strongly suggesting that painful hypersensitivity in FMS is produced by sensitization of peripheral nociceptors. In contrast, direct application of $\operatorname{IgG}$ to isolated DRG neurons was without effect on $\left[\mathrm{Ca}^{2+}\right]_{\mathrm{i}}$, consistent with an indirect action that may involve activity of an additional peripheral cell type, such as SGCs.

In combination with the electrophysiological observations, retention of FMS IgG in the DRG, the lack of $\operatorname{IgG}$ in the brain and spinal cord, and the in vivo binding of FMS IgG to SGCs and neurons strongly suggest that the pronociceptive actions of FMS IgG were driven by peripheral mechanisms. FMS IgG binding in human DRG and in vitro binding of FMS IgG to the surface of murine SGCs and neurons further emphasizes that the nociceptive effects induced by FMS IgG may stem from recognition of epitopes in the DRG. Increased signs of in vivo SGC activity imply that FMS IgG binding in the DRG has functional effects. It is clear that SGCs and sensory neurons can be electrically coupled and that both increased coupling and SGC-derived pronociceptive cytokines can influence neuronal excitability leading to hypersensitivity (56-58). Additionally, the accumulation of SGC- and neuron-bound FMS IgG may stimulate molecular changes in DRG-resident macrophages that contribute to neuronal hypersensitivity despite a lack of macrophage infiltration or proliferation. Furthermore, the absence of systemic increases in proinflammatory cytokines like TNF and IL-1 $\beta$ further supports our hypothesis that FMS IgG acts locally in the DRG, without inducing a systemic inflammatory response. Together, these findings point to the DRG as a site of action for FMS IgG and future studies will investigate the contributions of IgG acting on SGCs and neurons in driving pain-related sensory abnormalities and neuronal hypersensitivities in mice.

Although we did not discover a characteristic pattern of reactivities in patient sera in the protein microarray, we did note a substantial number of autoreactivities. Our results also indicated some enrichment of reactivities against proteins classified in GO terms for cellular components, and KEGG terms for pathways related to metabolism. Although the potential enrichment of reactivities may be encouraging, their relevance to the pathophysiology of FMS is currently unclear, since they were mostly detected in single samples. Furthermore, most proteins relevant to the potentially enriched terms are located intracellularly and are thus unlikely to be recognized by circulating IgG. Genuine autoantigens may also have escaped detection, since the linearized antigenic protein fragments used in the screen are unlikely to adopt their native conformation on the chip (47). The peptides and protein fragments were produced in E. coli, and therefore lack posttranslational modifications. The prevalence of reactivities against human peptides in our preliminary assessment of patient sera is nevertheless consistent with autoreactive $\operatorname{IgG}$ being responsible for our findings. Intriguingly, sera from COVID-19 patients contain a wide range of functional autoantibodies, which have been proposed to influence the symptomatic profile in patients (59).

Small-nerve-fiber pathology and a reduction in skin innervation has been observed in FMS patients, and is associated with a more severe disease burden and may contribute to peripheral sensitivity (33). Remarkably, we observed that transfer of FMS IgG to mice resulted in a reduction in IENFD in glabrous hind paw skin. In humans, it is still unclear why reduced skin innervation occurs, but our findings indicate that FMS IgG contributes to this fiber loss. FMS IgG may drive denervation from the DRG via SGC or neuronal cell body binding but may also act more distally through other mechanisms.

Future studies will examine the underlying cellular and molecular mechanisms responsible for FMS IgG-mediated peripheral sensitization. The possibility that the heightened noxious peripheral input produced by FMS IgG may generate altered patterns of activity in the central nervous system (19) also remains to be explored. Similarly, whether FMS IgG drives sensitivity to other sensory modalities, such as to auditory and olfactory stimuli, still needs to be investigated. The identification of a pivotal role for autoreactive IgG in the pathophysiology of FMS may transform future research and facilitate development of mechanism-based therapeutic interventions. Our results suggest that therapies which reduce the total IgG titer, such as plasmapheresis or immunoadsorption (e.g., with protein A columns), or which specifically 

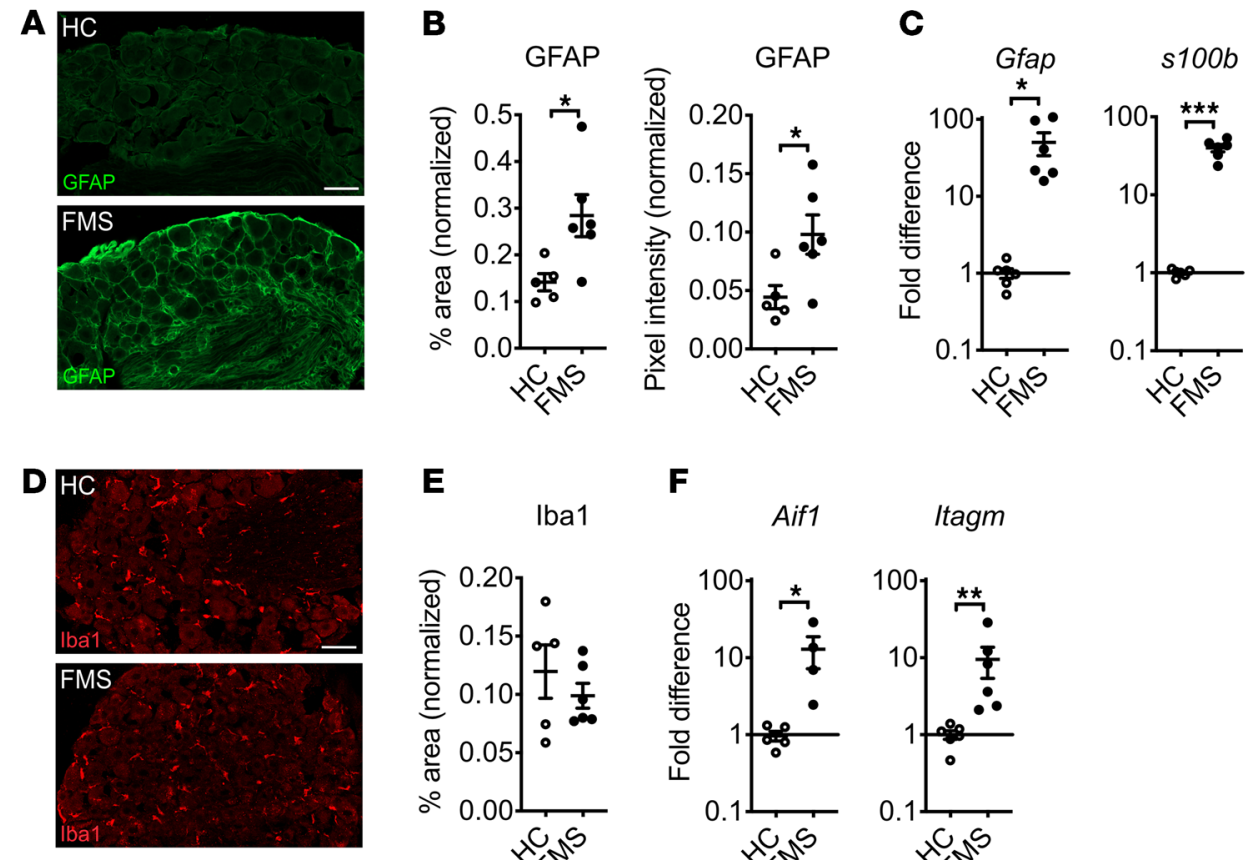

\section{E}

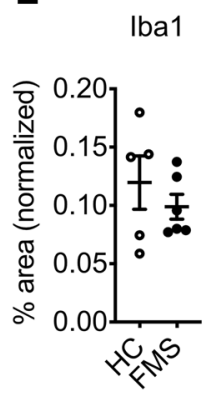

$\mathbf{F}$
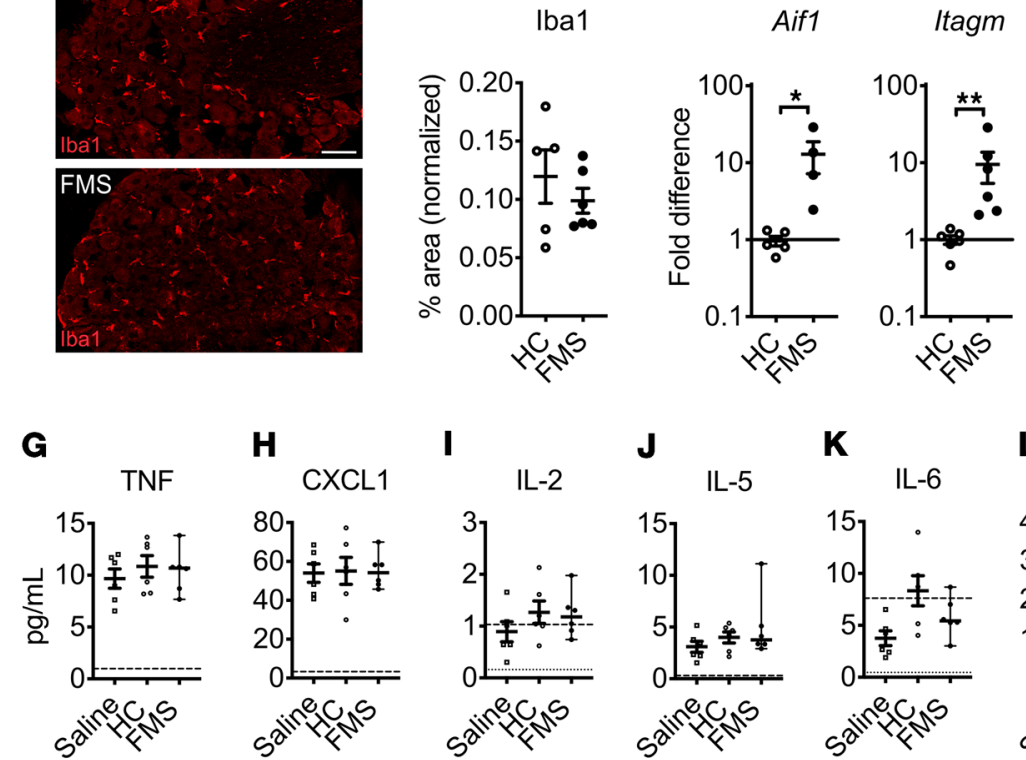

H I

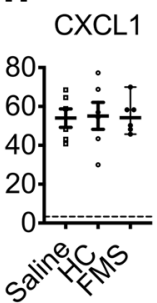

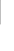

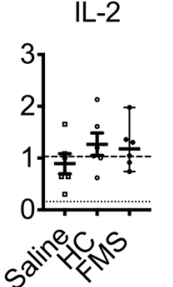

J

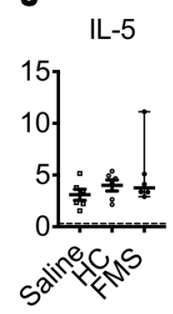

K

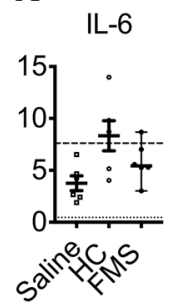

$\mathbf{L}$

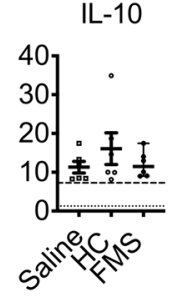

M

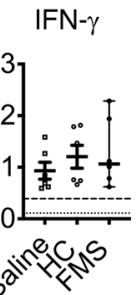

Figure 8. FMS IgC increases signs of satellite glial cell activity in vivo but does not drive systemic inflammation. DRG from FMS IgG-injected mice have increased GFAP immunoreactivity (A), which is indicative of increased satellite glial cell activity, compared with HC IgC injected mice when the percentage area of GFAP immunoreactivity and GFAP mean pixel intensity are quantified and normalized to the DAPI signal (B). Gfap and s100b gene expression is elevated in the DRG of mice injected with FMS IgC compared with HC IgG (C). The number of Iba1-immunoreactive macrophages was unchanged, as was the percentage area of Iba1 immunoreactivity, when comparing HC IgG- and FMS IgG-injected mice ( $\mathbf{D}$ and $\mathbf{E}$ ). Gene expression of Aif1 (Iba1 gene) and Itagm (gene for CD11b, another macrophage marker) was elevated in FMS IgG-injected mice compared with HC IgG-injected mice (F). Scale bars: $50 \mu \mathrm{m}$. qPCR data were normalized to Hprt1 expression analyzed using the $2^{-\Delta \Delta C t}$ method. Serum levels of TNF (G), CXCL1 (H), IL-2 (I), IL-5 (J), IL-6 (K), IL-10 (L), and IFN- $\gamma(\mathbf{M})$ were measured and there were no differences between groups. The dashed lines indicate the lower limit of quantification (LLOQ) and the dotted lines indicate the lower limit of detection (G-M). Line and whiskers indicate mean \pm SEM $(n=4-6)$. Differences between FMS IgG and HC IgG were analyzed with Mann-Whitney $U$ test (B-F). Cytokine levels were compared between saline, HC, and FMS with 1-way ANOVA followed by Tukey's post hoc test for each analyte (G-M) except IL-6, which was not analyzed statistically because most values were below the LLOQ. ${ }^{*} P<0.05,{ }^{* *} P<0.01,{ }^{* * *} P<0.001$.

reduce autoreactive IgG (using antigen-specific adsorption) may be effective for FMS (60). Alternatively, symptomatic therapies that interfere with the binding of autoreactive antibodies or prevent their functional consequences may also provide effective treatment approaches.

\section{Methods}

Study design. Our goal was to determine whether administration of IgG from FMS patients to mice transfers characteristic symptoms of FMS. FMS patients and age-matched $\mathrm{HC}$ individuals were recruited at 2 different sites, the University of Liverpool and the Karolinska Institute, where serum was collected and IgG was purified. First, we assessed whether IgG from FMS patients drove pain-like behavior in mice by transferring IgG from single individuals into mice as well as transferring IgG pooled from several individuals into mice. Second, we inves- tigated whether primary sensory afferents were sensitized following transfer of FMS or HC IgG into mice using an ex vivo skin-nerve preparation. Third, we examined FMS IgG localization following transfer and cell-type binding in vivo and in vitro with Western blotting and immunofluorescence. Last, we assessed IgG-induced changes in skin innervation using immunofluorescence. Mechanical hypersensitivity is a hallmark of FMS and the Randall-Selitto paw-pressure test is a sensitive translational measure of this symptom.

Power calculations indicated that $n=4$ would be sufficient to detect a 15-g reduction of the paw withdrawal threshold with $\alpha<0.025$ and $1-\beta>0.9$ (6 repeated measures, 2-sided), but $n=6$ to detect a reduction in the cold-plate paw withdrawal latency of 2.5 seconds. Electrophysiological experiments were analyzed using 1-sided tests since we examined whether the increased sensitivity observed in vivo was associated with an increased (not decreased) single unit respon- 
A
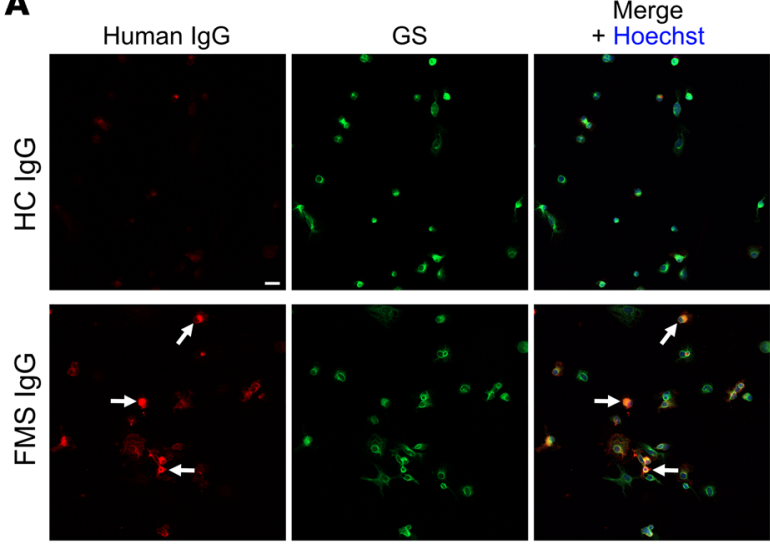

G
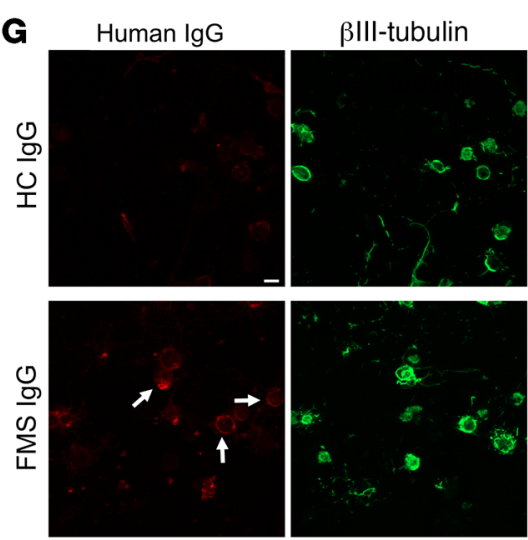

M
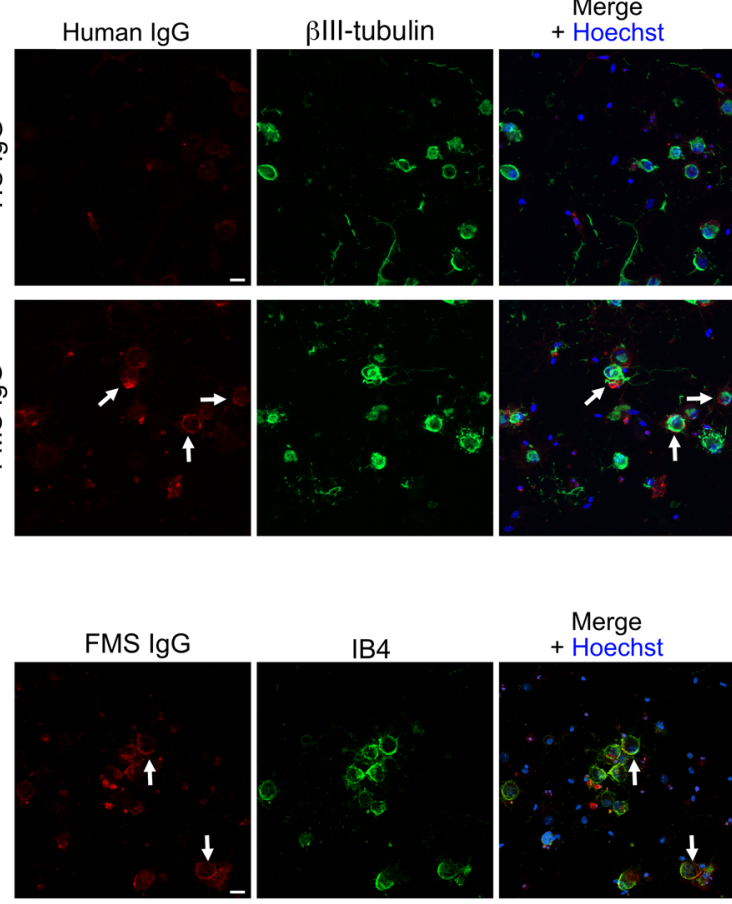

B

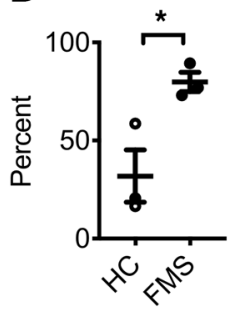

C

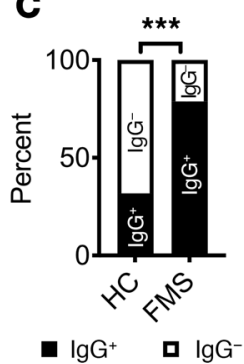

D

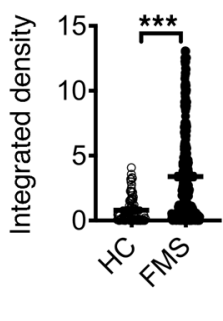

$\mathbf{E}$

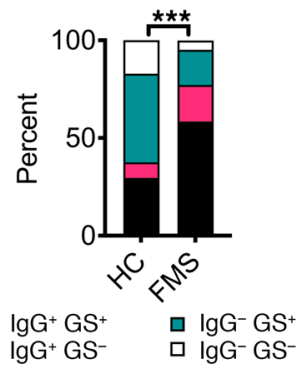

$\mathbf{F}$

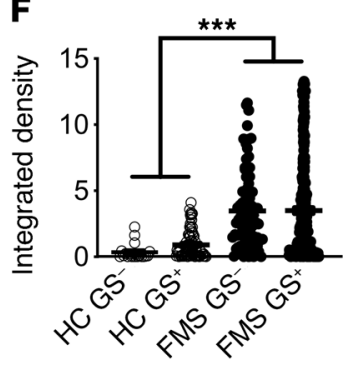

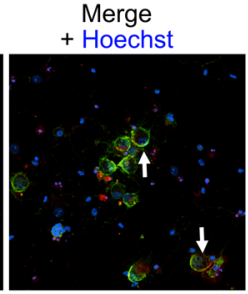

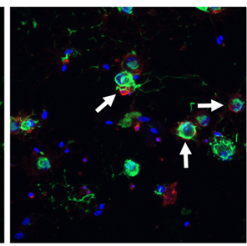

K

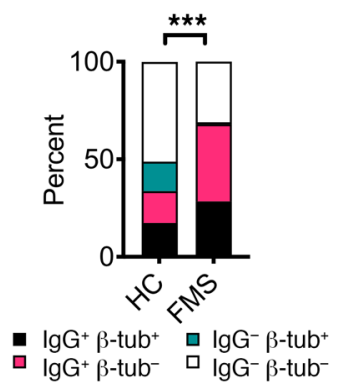

H

I
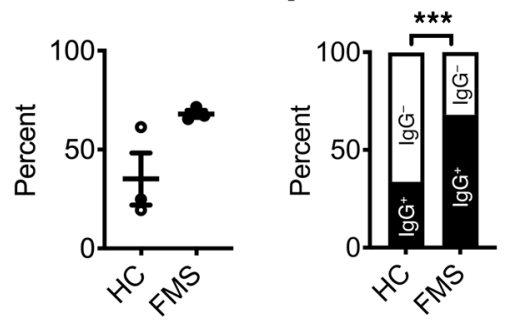

J

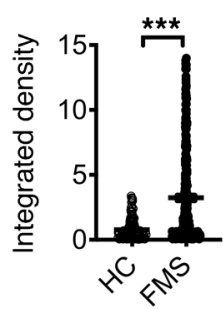

$\mathbf{L}$

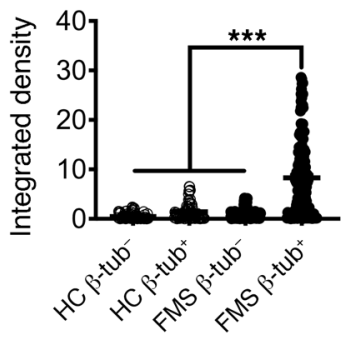

Figure 9. FMS IgG binds to satellite glial cells and to neurons in vitro. Live cells were incubated with FMS IgC or HC IgC to examine only cell surface binding. In satellite glial cell (SGC) cultures (A), FMS IgG labeled a greater percentage of cells than HC IgG when analyzed per animal (B) and by the total number cells (C). The immunoreactivity, analyzed as signal intensity (integrated density), was higher in cells incubated with FMS IgG than HC IgG (D). FMS IgC labeled SGCs (CS+) and non-SGCs (CS-) to a greater extent (percentage, E) and with a higher intensity (F) than HC IgC. FMS and HC IgC Iabeling of neuronal cultures (G) was not different when considering the percentage of cells labeled per animal (H), but a difference was observed when the total number of cells was considered (I). The signal intensity was higher for cells exposed to FMS IgC than HC IgG (J). FMS IgG Iabeled neurons ( $\beta$ III-tubulin+) and non-neurons ( $\beta$ III-tubulin ${ }^{-}$) to a greater extent than HC IgG (K). The signal intensity of FMS IgG binding to neurons was greater than FMS IgG binding to non-neuronal cells and HC IgG binding to all cells (L). FMS IgC and IB4 colocalization (M) indicates that FMS IgG binds neuronal cell membranes. All scale bars: $20 \mu \mathrm{m}$. Data points are the percentage of cells bound by HC or FMS IgC (B and $\mathbf{H})$. In $\mathbf{D}, \mathbf{F}, \mathbf{J}$, and $\mathbf{L}$ data points are the integrated density of individual cells across 3 experiments. Bar and whiskers indicate mean \pm SEM ( $n=3$ individual experiments). ${ }^{*} P<0.05 ;{ }^{* *} P<0.001$ by unpaired $t$ test (B, D, $\mathbf{H}$, and $\mathbf{~ )}$, $\chi^{2}$ test (C, E, I, and $\left.\mathbf{K}\right)$, or Kruskal-Wallis test with Dunn's post hoc test (F and $\left.\mathbf{L}\right)$.

siveness. All other data were analyzed using 2-sided tests. Behavioral (except the Comprehensive Animal Lab Monitoring System [CLAMS]) and electrophysiological experiments were performed at King's College London and CLAMS, immunofluorescence, Western blotting, qPCR, and cell culture studies were performed at the Karolinska Insti- tute. Immunofluorescence, Western blotting, and qPCR experiments were performed with tissues from behavioral experiments using IgG pools. Immunocytochemistry experiments were performed using IgG pools. Sample sizes for each experiment are additionally indicated in the figure legends. 
A
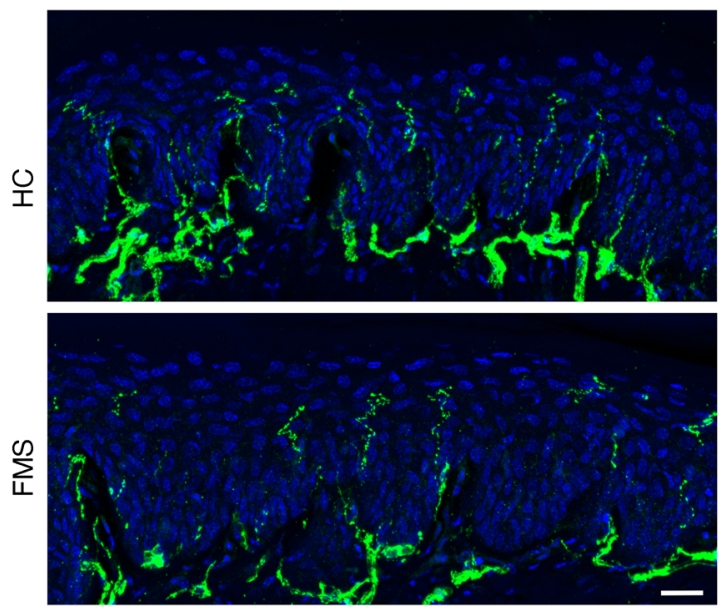

B

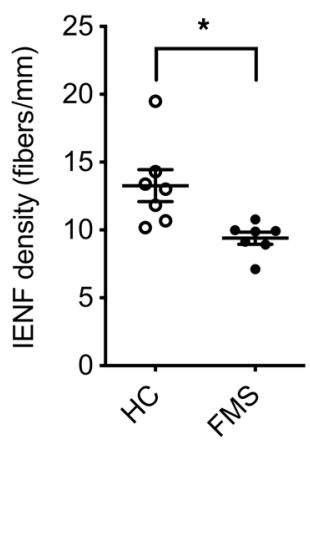

Figure 10. FMS IgG transfer decreases intraepidermal nerve fiber density. Intraepidermal nerve fibers (IENFs) were identified in the glabrous hind-paw skin with an anti-PGP 9.5 antibody (A). The number of IENFs crossing from the dermis to the epidermis was decreased following transfer of FMS IgC compared with $\mathrm{HC}$ IgC 14 days after the first injection (B) (pooled, $8 \mathrm{mg}$ per day for 4 consecutive days). Scale bar: $20 \mu \mathrm{m}$. Data points are mean $\pm \operatorname{SEM}(n=7)$. ${ }^{*} P$ $<0.05$ by unpaired $t$ test.

Patient samples. Serum samples for individual testing were derived from UK patients managed for their fibromyalgia at a department of pain medicine, or from age- and sex-matched HCs between April 2017 and November 2018. Pooled samples were obtained from Swedish patients between September 2015 and December 2016 or HCs responding to a study advert between March 2016 and March 2017. All patients had been examined by a consultant rheumatologist, and UK patients had additionally been examined by a consultant in pain medicine.

We purified serum IgG from 44 FMS patients and 39 HC subjects. All patients fulfilled both 1990 and 2011 ACR diagnostic criteria for fibromyalgia $(61,62)$. Most patients (42 of 44) and all of the pooled-sample donors were unaffected by other sensory, autoimmune, or rheumatological conditions. Most patient donors (43 of 44) were women. The donors' demographics and disease characteristics are provided in Supplemental Tables 1 and 2.

Purification of immunoglobulins. IgG for individual testing was purified as described previously (63), using protein G beads (SigmaAldrich). Serum was diluted 1:3 with Hartmann's solution, passed through a protein $\mathrm{G}$ column, and the bound IgG was eluted using 100 $\mathrm{mM}$ glycine $\mathrm{pH} 2.3$; after elution the $\mathrm{pH}$ was adjusted to 7.4 using $1 \mathrm{M}$ Tris pH 8 and then the eluate was dialyzed overnight at $4^{\circ} \mathrm{C}$ in Hartmann's using a $10 \mathrm{kDa}$ dialysis membrane (Thermo Fisher Scientific). The concentration of IgG present after dialysis was determined using a modified Lowry assay (DC protein assay, Bio-Rad) and adjusted by dilution with Hartmann's or by concentrating dialysis against a sucrose solution (Sigma-Aldrich). Finally, the IgG solution was sterile filtered using syringe-driven $0.2 \mu \mathrm{m}$ filter units (Millipore), stored at $4^{\circ} \mathrm{C}$, and used within 3 months. Sera for pooled sample testing were purified using HiTrap Protein G HP columns (GE Healthcare), eluted with $0.1 \mathrm{M}$ glycine/ $\mathrm{HCl} \mathrm{pH}$ 2.7, and the $\mathrm{pH}$ adjusted to 7.4 with $1 \mathrm{M}$ Tris $\mathrm{pH}$ 9; consequently, samples were dialyzed against PBS, concentration adjusted, stored at $-20^{\circ} \mathrm{C}$, and later thawed, pooled, and concentration-adapted using PBS-prewet concentration columns (Pall Corporation, Macrosep

10K); concentration measurements were performed using a Nanodrop 2000 (Thermo Fisher Scientific). Before eluting the IgG from the protein G column, the IgG-depleted sera (flow through) was collected and stored at $-80^{\circ} \mathrm{C}$ for later use. Prior to injection, the flow through was concentration-adapted using PBS-prewet concentration columns and the concentration was determined with a Nanodrop 2000.

Animals. Behavioral experiments were performed on female C57BL/6J mice (8-10 weeks old) obtained from Envigo UK Ltd and housed in a temperature-controlled environment with a 12-hour light/12-hour dark cycle with access to food and water ad libitum. Locomotor activity assessment and immunofluorescence experiments were performed on 4-month-old female BALB/c mice (Janvier). Mice were injected intraperitoneally with IgG from HC subjects or FMS patients on 1-4 consecutive days.

Behavioral studies. Before any nociceptive testing, mice were kept in their holding cages to acclimatize (10-15 minutes) to the experimental room. Mice were randomized between cages and the experimenter was blinded to their treatment.

The Randall-Selitto paw-pressure test was performed using an Analgesy-Meter (Ugo-Basile). The experimenter lightly restrained the mouse and applied a constantly increasing pressure stimulus to the dorsal surface of the hind paw using a blunt conical probe. The nociceptive threshold was defined as the force in grams at which the mouse withdrew its paw (64). A force cutoff value of $150 \mathrm{~g}$ was used to avoid tissue injury. The thigh-pressure test was also performed using an Analgesy-Meter. A constantly increasing pressure was applied to the inner thigh muscle of lightly restrained mice, using a blunt, wedgeshaped probe and the force at which the mouse withdrew its leg was recorded as the nociceptive threshold.

Tactile sensitivity was assessed using von Frey filaments (0.008-2 g) according to Chaplan's up-down method (65). Animals were placed in a Perspex chamber with a metal grid floor allowing access to their plantar surface and allowed to acclimatize prior to the start of the experiment. The von Frey filaments were applied to the plantar surface of the hind paw with enough force to allow the filament to bend, and held static for approximately 2-3 seconds. The stimulus was repeated up to 5 times at intervals of several seconds, allowing for resolution of any behavioral responses to previous stimuli. A positive response was noted if the paw was sharply withdrawn in response to filament application or if the mouse flinched upon removal of the filament. Any movement of the mouse, such as walking or grooming, was deemed an unclear response, and in such cases the stimulus was repeated. If no response was noted, a higher-force hair was tested and the filament producing a positive response recorded as the threshold.

Thermal sensitivity was assessed using a cold plate (Ugo Basile). Paw withdrawal latencies were determined with the plate set at $10^{\circ} \mathrm{C}$. The animals were lightly restrained (scruffed) and the left hind paw was placed onto the surface of the plate $(66,67)$. The latency to withdrawal of the paw was recorded as the endpoint. A maximum cutoff of 30 seconds was used for each paw.

Forelimb grip strength performance was assessed using a commercially available grip strength meter (Ugo Basile), incorporating a T-bar 
A
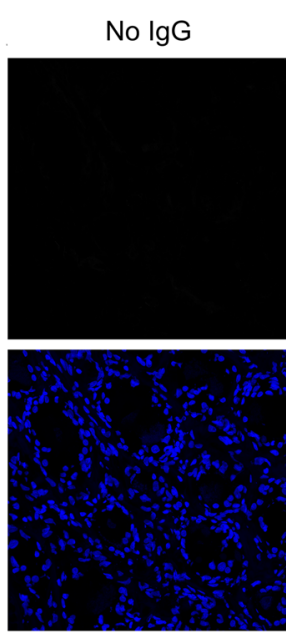

C
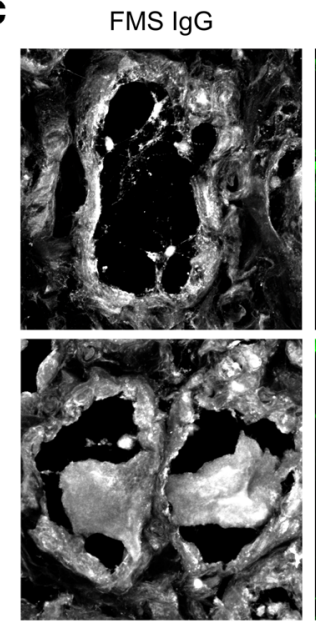

$\mathrm{HC} \lg \mathrm{G}$
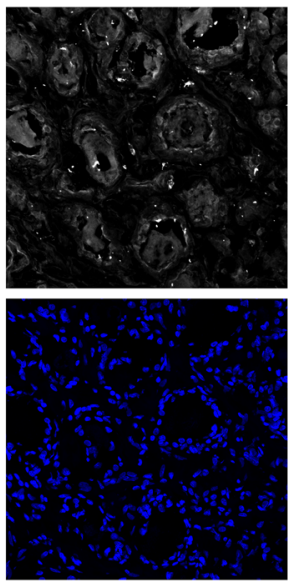

Indicated antibody
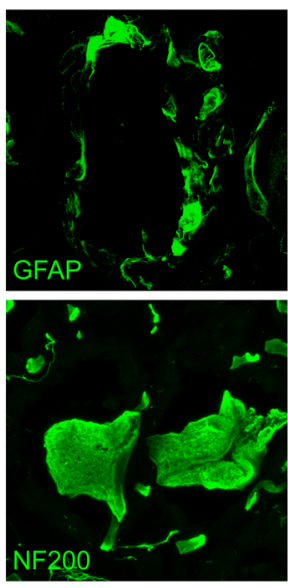

FMS IgG
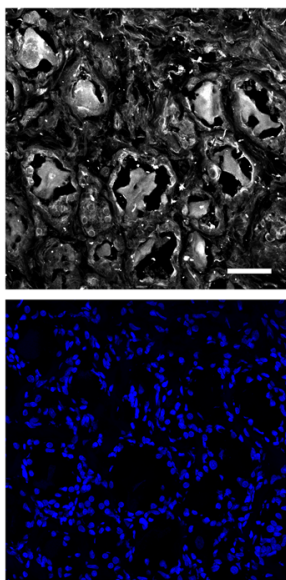

Merge
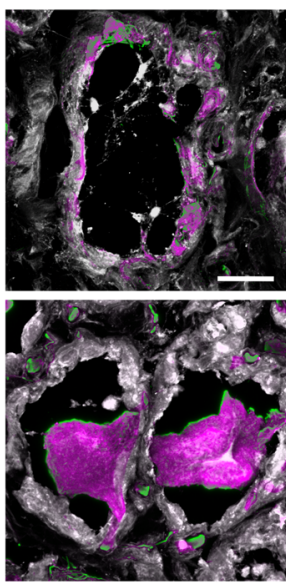

B

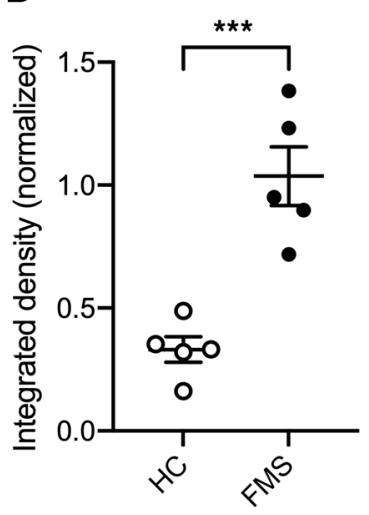

Figure 11. FMS IgG binds human DRG. FMS IgG bound human DRG tissue sections more intensely than HC IgG when assessed by integrated density normalized to DAPI (A and $\mathbf{B})$. The indicated IgG is in white in the top row and DAPI is in blue in the bottom row of A. High-magnification images of FMS IgG (white) demonstrate colocalization (purple) with GFAP-immunoreactive satellite glial cells (green) and NF-200-immunoreactive neurons (green) (C). Scale bars: $50 \mu \mathrm{m}$ (A) and $20 \mu \mathrm{m}$ (C). Data are mean \pm SEM ( $n=5$ independently stained slides). ${ }^{* *} P<0.001$ by unpaired $t$ test. and force transducer. The mice were held gently by the base of the tail and pulled across the horizontal T-bar so that they were able to grip the bar with their fore paws. A digital readout of the maximum force applied is given once the grip is released. Mice were acclimatized to the apparatus by testing twice prior to starting the study. The test was performed at least twice on each mouse and the mean force value used.

Locomotor activity was assessed with CLAMS (Columbus Instruments) cages for 48 hours. Mice were singly housed and habituated to the cages for 24 hours starting during the night 4 days following antibody injection. Following habituation, locomotor activity was assessed by beam-break recording. The CLAMS cage has a grid of infrared beams in the $x-y$ plane and records infrared beam breaks that result from the mouse moving in the cage. Beam breaks are counted and used to assess the total movement of the mouse over a 24 -hour period. Total movement is analyzed by the number of beam breaks (counts) in 20-minute bins over a 24-hour period. The night phase (peak activity phase) was further subdivided in to 4-hour bins from 18:00-6:00 hours.

Skin-nerve recording. Mice were killed by cervical dislocation and the hind paw was shaved prior to dissection of the isolated skin-nerve preparation. The saphenous nerve and the shaved skin of the hind limb were placed in a recording chamber at $32^{\circ} \mathrm{C}$. The chamber was perfused with a gassed $\left(95 \% \mathrm{O}_{2}\right.$ and $\left.5 \% \mathrm{CO}_{2}\right)$, prewarmed, synthetic interstitial fluid (SIF): $108 \mathrm{mM} \mathrm{NaCl}, 3.5 \mathrm{mM} \mathrm{KCl}, 0.7 \mathrm{mM} \mathrm{MgSO}_{4}$,
$26.2 \mathrm{mM} \mathrm{NaCO}_{3}, 1.65 \mathrm{mM} \mathrm{NaH}_{2} \mathrm{PO}_{4}, 1.53 \mathrm{mM} \mathrm{CaCl}_{2}, 9.6 \mathrm{mM}$ sodium gluconate, $5.55 \mathrm{mM}$ glucose, and $7.6 \mathrm{mM}$ sucrose. The skin was placed inside up (corium side up) and pinned down using insect pins ( $0.2 \mathrm{~mm}$ diameter) in the organ bath to allow access to the receptive fields. The saphenous nerve was threaded through a small gap from the organ bath to an adjacent recording chamber and positioned on a mirror platform. The desheathed saphenous nerve was covered with paraffin oil for electrical isolation and the dissected fine nerve filaments were visualized with a microscope and placed on a gold wire recording electrode $(42,43)$.

Conduction velocity. The saphenous nerve was divided into progressively thinner filaments until a single unit could be isolated in response to mechanical stimulation of the receptive field with a glass rod. The electrical latency of identified units (Digitimer DS2, Digitimer Ltd) was used to determine the conduction velocity and to categorize units as $\mathrm{A} \beta$ (velocity $>10 \mathrm{~m} / \mathrm{s}), \mathrm{A} \delta(1.2<$ velocity $<10 \mathrm{~m} / \mathrm{s}$ ), or C-fibers $(0<$ velocity $<1.2 \mathrm{~m} / \mathrm{s})$.

Mechanical and cold stimulation. A computer-controlled stimulating probe, equipped with a force transducer, was used to deliver mechanical stimuli to the most sensitive point of a receptive field (Avere Solutions UG) of AM and CM fibers in preparations from mice treated with FMS or HC IgG. Recording and analysis were done using Spike 2 (Cambridge Electronic Design). Cold sensitivity of CM units 
was investigated by isolating the receptive field with a metal ring and a 60 -second cold ramp $\left(\sim 31^{\circ} \mathrm{C}-4^{\circ} \mathrm{C}\right)$ was applied by superfusing precooled SIF. Peristaltic pumps were used to control the SIF flow rate. A digital temperature probe (GIA 2000, Greisinger) was placed inside the ring to record the temperature at the skin. A response was considered positive if a minimum of 2 action potentials were evoked and the temperature at which the second action potential fired was recorded as the cold threshold.

Immunofluorescence. Mice were deeply anesthetized with isoflurane and perfused with PBS, followed by $4 \%$ paraformaldehyde (PFA). The lumbar spinal cord, lumbar DRG, and hind paw glabrous skin were collected, postfixed for 24 hours in $4 \%$ PFA, cryoprotected in $20 \%$ sucrose, and then embedded in optimal cutting temperature (OCT) compound (Sakura Finetek). Tissue was sectioned with a CryoStar NX70 cryostat (Thermo Fisher Scientific) at a thickness of $10 \mu \mathrm{m}$ and thaw mounted onto SuperFrost Plus slides (Thermo Fisher Scientific) and slides were stored at $-20^{\circ} \mathrm{C}$ until use. Prior to use, slides were thawed for at least 30 minutes at room temperature and washed in PBS to remove excess OCT. Slides were blocked with PBS supplemented with $0.3 \%$ Triton X-100 and 3\% normal goat or donkey serum, depending on the secondary antibody, and then incubated with primary antibodies diluted in PBS supplemented with $0.1 \%$ Triton $\mathrm{X}-100$ and $1 \%$ normal goat or donkey serum. Slides were washed and incubated with appropriate secondary antibodies (indicated in Supplemental Table 4). For colocalization studies of human IgG with various cell-type markers the primary antibodies against the cell-type markers were first added, and then following washing, the anti-human IgG antibody was coincubated with the secondary antibodies against the cell-type marker primary antibodies. Following washing, the slides were incubated with DAPI (1:20,000), washed again, and then coverslipped with Prolong Gold mounting media (Thermo Fisher Scientific).

A human DRG was collected from a 53-year-old female without a history of chronic pain who died following a head trauma. The DRG was flash frozen at the time of collection and stored at $-80^{\circ} \mathrm{C}$ until it was embedded in OCT. Tissue was sectioned at a thickness of $14 \mu \mathrm{m}$ and mounted onto SuperFrost Plus slides and stored at $-80^{\circ} \mathrm{C}$ until use. For staining, sections were fixed for 30 minutes with cold $4 \%$ PFA, washed with PBS, blocked with PBS supplemented with $0.3 \%$ Triton X-100 and 3\% normal goat serum for 1 hour, and incubated overnight with $100 \mu \mathrm{g} / \mathrm{mL}$ unconjugated anti-human IgG Fab fragments (H+L, Jackson Immunoresearch). Following washing, slides were incubated overnight with pooled FM or HC $\operatorname{IgG}(15 \mu \mathrm{g} / \mathrm{mL})$ diluted in PBS with $1 \%$ normal goat serum and 0.1\% Triton X-100. Slides were then washed and incubated with appropriate secondary antibodies for 2 hours. Antibodies against NF200 or GFAP were applied for 2 hours, and then slides were washed and incubated with appropriate secondary antibodies. Following washing, the slides were incubated with DAPI (1:10,000), washed again, and then coverslipped with Prolong Gold mounting media.

Fluorescence microscopy and analysis. DRG were imaged using a confocal microscope (Zeiss LSM800) operated by LSM ZEN2012 (Zeiss) software. Human IgG accumulation and GFAP and Iba1 signal intensity were analyzed in Image (NIH) by defining a region of interest around the neuron-rich area of the DRG, applying a minimum pixel intensity threshold, and then the percentage positive area above the threshold and the average pixel intensity above the threshold were evaluated. Data were then normalized to the DAPI pixel inten- sity. Macrophage number was calculated by counting the number of Iba1-immunoreactive cells in the neuron-rich area of the DRG. IENFD was determined by counting the number of fibers crossing from the dermis to the epidermis. Spinal cords were imaged using a Nikon Eclipse TE300 fluorescence microscope with a Nikon Digital Sight DS-Fi1 camera. Spinal Iba1 and GFAP signal intensity was analyzed by 2 blinded reviewers using a custom-developed Python script. Data were then normalized to the DAPI pixel intensity. For DRG and spinal cords, 4- 5 sections from each animal, separated by at least 50 $\mu \mathrm{m}$, were analyzed and averaged for each experiment. For skin, 4 sections from each animal separated by at least $80 \mu \mathrm{m}$ were analyzed and averaged. Data are presented as mean \pm SEM. Representative spinal cord images were taken using a Zeiss LSM 800 confocal microscope.

Cell culture. DRG and SGC-enriched/neuron-depleted cultures were established using a modified protocol based on Malin et al. (68). Briefly, approximately 40 DRG were collected from each mouse and enzymatically digested in Papain (Worthington Biochemical Corp.) for 30 minutes and then in a Collagenase II (Worthington Biochemical Corp.)/Dispase (Sigma-Aldrich) mixture for 30 minutes. Following digestion, cells were suspended in F-12 media (Gibco) with $10 \%$ fetal bovine serum (FBS, Gibco) and 1× penicillin-streptomycin (Gibco). Gentle trituration was used to form a single-cell suspension. DRG cultures that were used for neuron staining were seeded into Nunc Lab-Tek II CC2-treated chamber slides (Thermo Fisher Scientific). SGC-enriched/neuron-depleted cultures were established by seeding cells into uncoated glass Nunc Lab-Tek chamber slides and 1.5 hours after seeding the media and nonadherent cells, including neurons, were removed; adherent cells were washed one time with fresh media, and fresh media were then added to the adherent cells. Cultures were kept in an incubator at $37^{\circ} \mathrm{C}$ with $5 \% \mathrm{CO}_{2}$. Cultures were allowed to recover for approximately 20 hours prior to immunocytochemistry or TUNEL staining.

Reagents. All buffers and salts were purchased from SigmaAldrich, VWR, or Thermo Fisher Scientific.

Statistics. Data are presented as individual data points or as mean \pm SEM, unless stated otherwise, and the number of animals or single units studied are indicated by $n$. Outliers in the immunofluorescence integrated density analysis were removed using the ROUT test; otherwise, no data points have been removed from any of the data sets presented. Data from behavioral in vivo experiments, most in vivo and in vitro immunofluorescence data, and qPCR data were assessed statistically by unpaired, 2-tailed $t$ test, Mann-Whitney test, 2-way repeated measure ANOVA followed by Sidak's or Dunnett's post hoc test, or 1-way ANOVA followed by Tukey's post hoc test. Patient data and electrophysiological data were evaluated by Mann-Whitney $U$ test. Differences in the distribution of cells bound by FMS or HC IgG were assessed by the $\chi^{2}$ test. Comparisons with a $P$ value of less than 0.05 were considered statistically different.

Study approval. Serum collection received ethical approval (UK: Ethics North-West Haydock, ref. 15/NW/0467; Sweden: approved by the local ethical committee 2011/2036-31/1) and samples were collected from individuals following written informed consent. A human DRG was collected from an organ donor following consent from the next of kin and received approval by the institutional review board (McGill University Health Centre REB 2019-4896). Behavioral experiments were carried out according to the UK Home Office Animal Procedures (1986) Act. All procedures in the UK were approved by the 
King's College London Animal Welfare and Ethical Review Body and conducted under a UK Home Office Project License. Animal experiments in Sweden were approved by the Stockholm North Animal Ethics Board (4945-2018).

\section{Author contributions}

AG conceived and initiated the UK studies, designed and conducted the Liverpool clinical aspects, and contributed to experimental design and to writing the manuscript. E Krock conceived, designed, performed, and analyzed cell culture experiments, undertook blood cytokine and IgG measurements, analyzed IgG binding, mRNA, and protein of mouse DRG, and wrote the manuscript. CG designed, performed, and analyzed behavioral experiments. MRI designed, performed, and analyzed electrophysiological experiments and contributed to writing the manuscript. AJ performed and analyzed cell culture experiments, analyzed immunohistochemical experiments, and assisted in patient sample processing. CMU designed and performed experiments for immunohistochemical analysis of mouse skin and human DRG. KS performed and analyzed behavioral and immunohistochemical experiments and assisted in patient sample processing. NV designed, performed, and analyzed electrophysiological experiments. MM performed and analyzed experiments and contributed to writing the manuscript. UC performed and analyzed electrophysiological experiments. SS performed Liverpool trial sample preparations and contributed to writing the manuscript. YN performed and analyzed cell culture experiments. JM performed and analyzed cell culture and mouse experiments, DRG immunohistochemical experiments, and purified IgG from patient samples. AB purified IgG from patient samples. LB performed and analyzed immunohistochemical experiments. AS examined the Stockholm cohort, including quantitative sensory testing. JT screened healthy controls in the Stockholm cohort and examined the Stockholm cohort, including quantitative sensory testing and blood sampling. DK performed screening and diagnosis of the Stockholm cohort and blood sampling. LH coordinated human DRG collection from organ donors. E Kosek designed and coordinated the clinical aspects of the study in Stockholm, and contributed to writing the clinical parts of the manuscript. CIS initiated the Swedish studies and SB, CIS, and DAA conceived the studies, designed and analyzed experiments, and wrote the manuscript. All authors participated in the critical reading of the manuscript and gave their consent for the final draft. The order of the co-first authors, AG and E Krock, was determined alphabetically.

\section{Acknowledgments}

This work was supported by the Medical Research Council grant MR/S003428/1 (to DAA, SB, and AG), Versus Arthritis grants 21544 (to DAA and AG) and 21543 (to SB), the Pain Relief Foundation Liverpool (to DAA, SS, and AG), Eli Lilly \& Co (to DAA), Swedish Research Council grant 542-2013-8373 (to CIS), the Knut and Alice Wallenberg Foundation (018.0161 to CIS), the Karolinska Institute Foundations (to CMU), the European Union Seventh Framework Programme (FP7/2007-2013) under grant agreement number 602919 (to CIS and E Kosek), European Union's Horizon 2020 research and innovation programme under the Marie Skłodowska-Curie Grant Agreement number 764860 (to CIS and E Kosek), the European Research Council (ERC) under the European Union's Horizon 2020 research and innovation programme under the grant agreement no. 866075 (to CIS), by a generous donation from Leif Lundblad and family (to CIS and E Kosek), Stockholm County Council (grant ALF-20190039 to E Kosek), the IASP John J. Bonica fellowship (to E Krock), a CIHR postdoctoral fellowship (MFE-171299 to E Krock), and the Konung Gustaf V:s 80-årsfond (to E Krock). The authors are grateful to Domenico Cozzetto from the Translational Bioinformatics Platform at the NIHR Biomedical Research Centre at Guy's and St. Thomas's NHS Foundation Trust and King's College London for bioinformatics advice. The graphical abstract was created with BioRender and the Strategic Research Programme in Diabetes at Karolinska Institutet provided access to the CLAMS system.

Address correspondence to: David A. Andersson or Stuart Bevan, Wolfson CARD, Hodgkin Building, Guy's Campus, London SE1 1UL, United Kingdom. Phone: 44.207.848.6141; Email: david. andersson@kcl.ac.uk (DAA); Email: stuart.bevan@kcl.ac.uk (SB). Or to: Andreas Goebel, Pain Research Institute, Clinical Sciences Centre, Lower Lane, Liverpool L9 7AL, United Kingdom. Phone 44.151.529.5835; Email: andreasgoebel@rocketmail. com. Or to: Camilla I. Svensson, Center for Molecular Medicine L8:03, Visionsgatan 18, 17176 Stockholm, Sweden. Phone: 46.8.524.87948; Email: camilla.svensson@ki.se.
1. Kosek E, et al. Sensory dysfunction in fibromyalgia patients with implications for pathogenic mechanisms. Pain. 1996;68(2-3):375-383.

2. Hurtig IM, et al. Quantitative sensory testing in fibromyalgia patients and in healthy subjects: identification of subgroups. Clin J Pain. 2001;17(4):316-322.

3. Blumenstiel K, et al. Quantitative sensory testing profiles in chronic back pain are distinct from those in fibromyalgia. Clin J Pain. 2011;27(8):682-690.

4. Berwick RJ, et al. A systematic review into the influence of temperature on fibromyalgia pain: meteorological studies and quantitative sensory testing [published online January 6, 2021]. J Pain. https://doi.org/10.1016/j.jpain.2020.12.005.

5. Arnold LM, et al. Comorbidity of fibromyalgia and psychiatric disorders. JClin Psychiatry. 2006;67(8):1219-1225.

6. Thieme K, et al. Comorbid depression and anxiety in fibromyalgia syndrome: relationship to somatic and psychosocial variables. Psychosom Med. 2004;66(6):837-844.

7. Clauw DJ. Fibromyalgia: a clinical review. JAMA. 2014;311(15):1547-1555.

8. Queiroz LP. Worldwide epidemiology of fibromyalgia. Curr Pain Headache Rep. 2013;17(8):356.

9. Perrot $S$, et al. Patient phenotypes in fibromyalgia comorbid with systemic sclerosis or rheumatoid arthritis: influence of diagnostic and screening tests. Screening with the FiRST questionnaire, diagnosis with the ACR 1990 and revised ACR 2010 criteria. Clin Exp Rheumatol. 2017;35 suppl 105(3):35-42.
10. Fitzcharles M-A, et al. Comorbid fibromyalgia: a qualitative review of prevalence and importance. Eur J Pain. 2018;22(9):1565-1576.

11. Sluka KA, Clauw DJ. Neurobiology of fibromyalgia and chronic widespread pain. Neuroscience. 2016;338:114-129.

12. Macfarlane GJ, et al. EULAR revised recommendations for the management of fibromyalgia. Ann Rheum Dis. 2017;76(2):318-328.

13. Mascarenhas RO, et al. Association of therapies with reduced pain and improved quality of life in patients with fibromyalgia: a systematic review and meta-analysis. JAMA Intern Med. 2021;181(1):104-112.

14. Yen L-T, et al. Targeting ASIC3 for relieving mice fibromyalgia pain: roles of electroacupuncture, opioid, and adenosine. Sci Rep. 2017;7:46663. 
15. Nagakura Y, et al. Biogenic amine depletion causes chronic muscular pain and tactile allodynia accompanied by depression: a putative animal model of fibromyalgia. Pain. 2009;146(1-2):26-33.

16. Berglund B, et al. Quantitative and qualitative perceptual analysis of cold dysesthesia and hyperalgesia in fibromyalgia. Pain. 2002;96(1-2):177-187.

17. Kosek E, Hansson P. Modulatory influence on somatosensory perception from vibration and heterotopic noxious conditioning stimulation (HNCS) in fibromyalgia patients and healthy subjects. Pain. 1997;70 (1):41-51.

18. Jensen $\mathrm{KB}$, et al. Evidence of dysfunctional pain inhibition in fibromyalgia reflected in rACC during provoked pain. Pain. 2009;144(1-2):95-100.

19. Jensen KB, et al. Overlapping structural and functional brain changes in patients with long-term exposure to fibromyalgia pain. Arthritis Rheum. 2013;65(12):3293-3303.

20. Schrepf A, et al. Endogenous opioidergic dysregulation of pain in fibromyalgia: a PET and fMRI study. Pain. 2016;157(10):2217-2225.

21. Kaplan CM, et al. Functional and neurochemical disruptions of brain hub topology in chronic pain. Pain. 2019;160(4):973-983.

22. Serra J, et al. Hyperexcitable $\mathrm{C}$ nociceptors in fibromyalgia. Ann Neurol. 2014;75(2):196-208.

23. Üçeyler N, et al. Small fibre pathology in patients with fibromyalgia syndrome. Brain. 2013;136 (pt 6):1857-1867.

24. Uçeyler N, et al. Systematic review with meta-analysis: cytokines in fibromyalgia syndrome. BMC Musculoskelet Disord. 2011;12:245.

25. Andrés-Rodríguez L, et al. Peripheral immune aberrations in fibromyalgia: a systematic review, meta-analysis and meta-regression. Brain Behav Immun. 2020;87:881-889.

26. Tékus V, et al. A CRPS-IgG-transfer-trauma model reproducing inflammatory and positive sensory signs associated with complex regional pain syndrome. Pain. 2014;155(2):299-308.

27. Li W-W, et al. Autoimmunity contributes to nociceptive sensitization in a mouse model of complex regional pain syndrome. Pain. 2014;155(11):2377-2389.

28. Dawes JM, et al. Immune or genetic-mediated disruption of CASPR 2 causes pain hypersensitivity due to enhanced primary afferent excitability. Neuron. 2018;97(4):806-822.

29. Toyka KV, et al. Myasthenia gravis: passive transfer from man to mouse. Science. 1975;190(4212):397-399.

30. Toyka KV, et al. Myasthenia gravis. Study of humoral immune mechanisms by passive transfer to mice. N Engl J Med.1977;296(3):125-131.

31. Cuhadar U, et al. Autoantibodies produce pain in complex regional pain syndrome by sensitizing nociceptors. Pain. 2019;160(12):2855-2865.

32. Staud R, et al. Body pain area and painrelated negative affect predict clinical pain intensity in patients with fibromyalgia. J Pain. 2004;5(6):338-343.

33. Evdokimov D, et al. Reduction of skin inner- vation is associated with a severe fibromyalgia phenotype. Ann Neurol. 2019;86(4):504-516.

34. Aparicio VA, et al. Handgrip strength in men with fibromyalgia. Clin Exp Rheumatol. 2010;28(6 suppl 63):S78-S81.

35. Salaffi F, et al. Force-time curve features of handgrip strength in fibromyalgia syndrome. Sci Rep. 2020;10(1):3372.

36. French MA, Harrison G. Serum IgG subclass concentrations in healthy adults: a study using monoclonal antisera. Clin Exp Immunol. 1984;56(2):473-475

37. Unverdorben F, et al. Pharmacokinetic properties of $\mathrm{IgG}$ and various Fc fusion proteins in mice. MAbs. 2016;8(1):120-128.

38. Wolfe F, et al. The American College of Rheumatology preliminary diagnostic criteria for fibromyalgia and measurement of symptom severity. Arthritis Care Res (Hoboken). 2010;62(5):600-610.

39. Jaime-Lara RB, et al. A qualitative metasynthesis of the experience of fatigue across five chronic conditions. J Pain Symptom Manage. 2020;59(6):1320-1343.

40. Raftery G, et al. Are fibromyalgia patients as inactive as they say they are? Clin Rheumatol. 2009;28(6):711-714.

41. Bennett RM, et al. Aerobic fitness in patients with fibrositis. A controlled study of respiratory gas exchange and 133xenon clearance from exercising muscle. Arthritis Rheum. 1989;32(4):454-460.

42. Zimmermann $\mathrm{K}$, et al. Phenotyping sensory nerve endings in vitro in the mouse. Nat Protoc. 2009;4(2):174-196.

43. Vastani N, et al. Impaired nociception in the diabetic Ins $2^{+/ A k i t a}$ mouse. Diabetes. 2018;67(8):1650-1662.

44. Dalmau J, et al. Autoantibodies to synaptic receptors and neuronal cell surface proteins in autoimmune diseases of the central nervous system. Physiol Rev. 2017;97(2):839-887.

45. Oaklander AL, et al. Objective evidence that small-fiber polyneuropathy underlies some illnesses currently labeled as fibromyalgia. Pain. 2013;154(11):2310-2316.

46. Uhlén M, et al. Proteomics. Tissue-based map of the human proteome. Science. 2015;347(6220):260419.

47. Sjöberg R, et al. Exploration of high-density protein microarrays for antibody validation and autoimmunity profiling. N Biotechnol. 2016;33(5 pt A):582-592.

48. Liao Y, et al. WebGestalt 2019: gene set analysis toolkit with revamped UIs and APIs. Nucleic Acids Res. 2019;47(W1):W199-W205.

49. McMahon SB, et al. Crosstalk between the nociceptive and immune systems in host defence and disease. Nat Rev Neurosci. 2015;16(7):389-402.

50. Goebel A. Autoantibody pain. Autoimmun Rev. 2016;15(6):552-557.

51. Helyes Z, et al. Transfer of complex regional pain syndrome to mice via human autoantibodies is mediated by interleukin-1-induced mechanisms. Proc Natl Acad Sci U S A. 2019;116(26):13067-13076.
52. Albrecht DS, et al. Brain glial activation in fibromyalgia - a multi-site positron emission tomography investigation. Brain Behav Immun. 2019;75:72-83.

53. O'Brien AT, et al. Defective endogenous pain modulation in fibromyalgia: a meta-analysis of temporal summation and conditioned pain modulation paradigms. J Pain. 2018;19(8):819-836.

54. Wilbarger JL, Cook DB. Multisensory hypersensitivity in women with fibromyalgia: implications for well being and intervention. Arch Phys Med Rehabil. 2011;92(4):653-656.

55. Geisser ME, et al. A psychophysical study of auditory and pressure sensitivity in patients with fibromyalgia and healthy controls. J Pain. 2008;9(5):417-422.

56. Spray DC, et al. Gap junction mediated signaling between satellite glia and neurons in trigeminal ganglia. Glia. 2019;67(5):791-801.

57. Ji R-R, et al. Glia and pain: is chronic pain a gliopathy? Pain. 2013;154 suppl 1(0 1):S10-S28.

58. Xu M, et al. Pain and the immune system: emerging concepts of IgG-mediated autoimmune pain and immunotherapies. J Neurol Neurosurg Psychiatry. 2020;91(2):177-188.

59. Wang EY, et al. Diverse functional autoantibodies in patients with COVID-19 [preprint]. https://doi. org/10.1101/2020.12.10.20247205. Posted on medRxiv December 12, 2020.

60. Schwartz J, et al. Guidelines on the use of therapeutic apheresis in clinical practiceevidence-based approach from the writing committee of the American Society for Apheresis: the seventh special issue. J Clin Apher. 2016;31(3):149-162.

61. Wolfe F, et al. The American College of Rheumatology 1990 criteria for the classification of fibromyalgia. Report of the multicenter criteria committee. Arthritis Rheum. 1990;33(2):160-172.

62. Wolfe F, et al. Fibromyalgia criteria and severity scales for clinical and epidemiological studies: a modification of the ACR preliminary diagnostic criteria for fibromyalgia. J Rheumatol. 2011;38(6):1113-1122.

63. Goebel A, et al. Intravenous immunoglobulin response and evidence for pathogenic antibodies in a case of complex regional pain syndrome 1 . Ann Neurol. 2005;57(3):463-464

64. Randall LO, Selitto JJ. A method for measurement of analgesic activity on inflamed tissue. Arch Int Pharmacodyn Ther. 1957;111(4):409-419.

65. Chaplan SR, et al. Quantitative assessment of tactile allodynia in the rat paw. J Neurosci Methods. 1994;53(1):55-63.

66. Gentry C, et al. The roles of iPLA2, TRPM8 and TRPA1 in chemically induced cold hypersensitivity. Mol Pain. 2010;6:4.

67. Andersson DA, et al. Clioquinol and pyrithione activate TRPA1 by increasing intracellular $\mathrm{Zn}^{2+}$. Proc Natl Acad Sci U S A. 2009;106(20):8374-8379.

68. Malin SA, et al. Production of dissociated sensory neuron cultures and considerations for their use in studying neuronal function and plasticity. Nat Protoc. 2007;2(1):152-160. 